\title{
Point-contact Andreev-reflection spectroscopy of doped manganites: Charge carrier spin-polarization and proximity effects
}

\author{
(Review Article)
}

\author{
V.N. Krivoruchko, A.I. D'yachenko, and V.Yu. Tarenkov \\ A.A. Galkin Donetsk Physics and Technology Institute National Academy of Sciences of the Ukraine \\ 72 R. Luxemburg Str., Donetsk 83114, Ukraine \\ E-mail: krivoruc@gmail.com \\ Received September 18, 2012
}

\begin{abstract}
Materials with spin-polarized charge carriers are the most demanded in the spin-electronics. Particularly requested are the so-called half-metals which have the maximum attainable value of carrier spin polarization. Doped manganites are in the list of compounds with, potentially, half-metallic properties. The point-contact (PC) Andreevreflection (AR) spectroscopy is a robust and direct method to measure the degree of current spin polarization. In this report, advances in PCAR spectroscopy of ferromagnetic manganites are reviewed. The experimental results obtained on "classic" s-wave superconductor - ferromagnetic manganites PCs, as well as related theoretical models applied to deduce the actual value of charge carrier spin-polarization, are discussed. Data obtained on "proximity affected" contacts is also outlined. Systematic and repeatable nature of a number of principal experimental facts detected in the AR spectrum of proximity affected contacts suggests that some new physical phenomena have been documented here. Different models of current flow through a superconductor-half-metal ferromagnet interface, as well as possibility of unconventional superconducting proximity effect, have been discussed.
\end{abstract}

PACS: $72.25 . \mathrm{Mk}$ Spin transport through interfaces;

74.45.+c Proximity effects; Andreev reflection; SN and SNS junctions;

72.25.Ba Spin-polarized transport in metals.

Keywords: current spin polarization, Andreev-reflection spectroscopy, ferromagnetic manganites, unconventional pairing.

\section{Contents}

1. Introduction............277

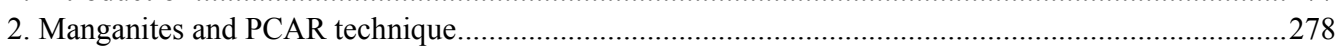

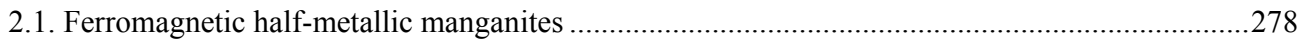

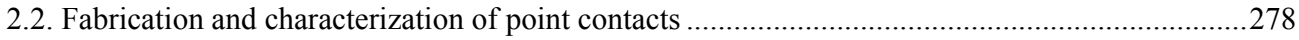

3. Theoretical basis of the AR spectroscopy of current spin polarization ...............................................279

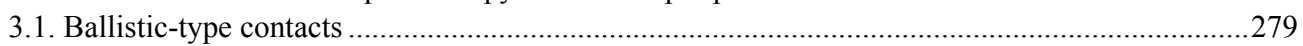

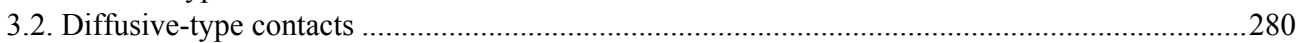

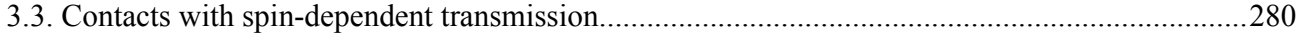

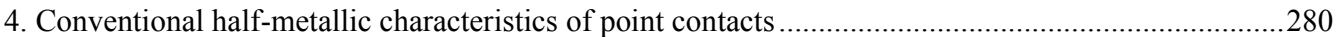

5. Proximity affected contacts, "anomalous" Andreev reflection...........................................................282

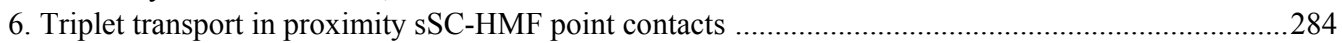

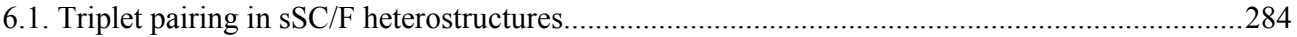

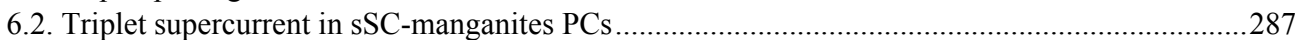

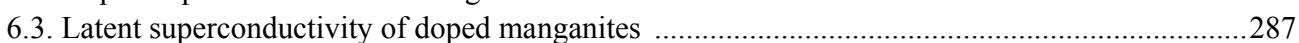

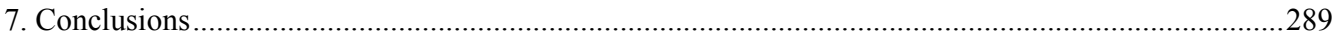

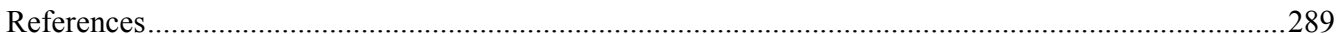




\section{Introduction}

Spintronics is a research field wherein two fundamental branches of physics - magnetism and electronics - are combined [1-4], and is based on the opportunity of ferromagnetic materials to provide spin-polarized current. The performance of many spintronics devices improves dramatically as the degree of charger carriers spin polarization, $P_{C}$, increases, and materials with highly spinpolarized current are of crucial importance here. Particular attention has focused on the so-called "half-metals". Halfmetals are ferromagnets having a Fermi surface in one spin band, and a gap for the opposite spin direction; i.e., halfmetals have the maximum attainable value of spin polarization $\left(P_{C}=100 \%\right)$. Yet, measuring $P_{C}$ is not an easy task. Almost all methods of measuring it necessitate removing electrons from the material under investigation. The densities of quasiparticle states at the Fermi level $E_{F}$ must therefore be weighted by their probability of escape, which generally depends on the measuring method and is also different for electrons with up $(\uparrow)$ and down $(\downarrow)$ spin.

The degree of spin polarization can be defined in several different ways. Methods available to measure spin polarization include spin-polarized photoemission [5], and transport measurements in point contacts and tunnel junctions with two ferromagnetic electrodes (see, e.g., [6]), or with ferromagnetic and superconducting electrodes. The latter case splits into two spectroscopic methods: the tunneling spectroscopy, originated in Tedrow-Meservey works on probing spin polarization [7], and the point-contact (PC) Andreev-reflection (AR) spectroscopy based on the way of supercurrent conversion into quasiparticle current at superconductor (SC)-normal metal $(\mathrm{N})$ interface invented by Andreev [8].

The conventional technique of spin-resolved photoemission [5] measures the spin of the electrons emitted from a region close to the surface of a ferromagnet of order of 5-10 $\AA$, and thus is quite surface-sensitive. The Tedrow and Meservey [7] method requires the material under investigation to be fabricated as part of a ferromagnet/superconductor tunnel junction, in which the superconducting density of states is then Zeeman split by the application of a magnetic field of several Tesla. The PC method offers several apparent advantages compared to these techniques. There are no restrictions on the sample geometry and one can avoid complex fabrication steps. In addition, it has excellent energy resolution, typically about $0.1 \mathrm{meV}$, and does not necessarily require an applied magnetic field. This spectroscopic method was invented over 35 years ago by I.K. Yanson [9] as an experimental tool to investigate the interaction mechanisms between electrons and phonons in metals. The PC technique was later used to study all kinds of scattering of electrons by elementary excitation in metals [10-12]. If one of the sides of a PC is a SC, the PC's differential conductance contains fundamental information on the excitation spectrum of quasiparticles. For this reason PC spectroscopy has become an important, sometimes unique, tool for investigation of superconductors (see, e.g., recent review [13] and references therein).

The PCAR spectroscopy is a technique in which the differential conductance $G=d I / d V$ is measured for an electrical PC with little or no tunneling barrier established between the superconducting tip and the counter-electrode (or vice versa). The idea that $\mathrm{AR}$ at the interface between a ferromagnet (F) and a SC can be used for direct probing of current spin polarization was apparently suggested for the first time by de Jong and Beenakker [14]. The idea is simple: the Andreev reflection can be visualized as two currents of electrons with opposite spins flowing inside a normal metal towards its interface with a SC. At the interface (more precisely, within the coherence length from the interface) the two currents recombine creating the current of singlet Cooper pairs. In a nonmagnetic metal both spin subband currents are the same, so in the superconducting state one can observe a $100 \%$ increase in the net current over the normal state. It was suggested [14] that in a ferromagnetic metal the total Andreev current is defined by that spin channel where the normal-state current is smaller, because the excess electrons in the other channel will not find partners to form Cooper pairs with.

The success of the first experiments for measuring charge carrier's spin polarization $[15,16]$ with a direct superconductor $\mathrm{PC}$ to an F established AR as a useful tool for characterizing materials for spin-electronics applications. Since then, this technique has been used to measure spin polarization in a broad range of ferromagnetic materials, including the transition metal elements $\mathrm{Fe}, \mathrm{Ni}$, and $\mathrm{Co}$ $[15,17]$, metallic alloys of transition metals such as permalloy $[15,18]$, Heusler alloys such as NiMnSb [15,19], halfmetals such as $\mathrm{CrO}_{2}[15,19-21]$, doped manganites [15,19, 22-29], and other ferromagnetic metals and semiconductors. The foregoing may lead to a conclusion that the PCAR technique is indeed a robust and universal way for measuring spin polarization applicable to all magnetic compounds. There are, however, a few cases when this technique can not be applied. For example, magnetite, $\mathrm{Fe}_{3} \mathrm{O}_{4}$, is ferrimagnet with anomalously high Curie temperature $\sim 850 \mathrm{~K}$. The band-structure calculations (see, e.g., [30]) have predicted that $\mathrm{Fe}_{3} \mathrm{O}_{4}$ is a half-metal with only one spin subband at the Fermi level. Experimentally, the spin-resolved photoemission on epitaxial thin films indeed indicated a spin polarization about $80 \%$ at room temperature [31] However, below a Verwey transition at about $120 \mathrm{~K}$, single crystal $\mathrm{Fe}_{3} \mathrm{O}_{4}$ shows a nonmetallic (polaronic) type of conductivity (see, e.g., [32]), and, in fact, the PCAR method cannot be used.

The objective of the report is to give a short review of experiments exploring charge carrier's spin-polarization in half-metallic manganites by the PCAR technique. We start, Sec. 2, with brief review of physics of ferromagnetic man- 
ganites as materials with half-metallic band structure. Then we discuss major issues concerning the fabrication and characterization of point contacts and possible related imperfections. Section 3 is devoted to the theoretical basis for the PCAR spectroscopy of current spin polarization. The models of point contacts with different character of conduction (ballistic, diffusive, etc.) are discussed. The following three sections are central. Firstly, we overview the experimental results obtained for conventional PCs of $s$-wave SC-manganite and estimate the degree of charge carrier's spin polarization for doped manganites (Sec. 4). Then, in Sec. 5, we discuss the results obtained for the socalled "proximity affected" contacts for which a few unconventional features have been detected. A possible physical scenario explaining the observations is discussed in Sec. 6. We end with summary and conclusions.

\section{Manganites and PCAR technique}

\subsection{Ferromagnetic half-metallic manganites}

In doped manganites, the ferromagnetic ordering has been attributed to the double-exchange interaction between the valence electronic states of $\mathrm{Mn}^{3+}-\mathrm{O}^{2-}-\mathrm{Mn}^{4+}$ bonds. Within the double-exchange model [33-36] the itinerant charge carriers provide both the magnetic interaction between nearest $\mathrm{Mn}^{3+}-\mathrm{Mn}^{4+}$ ions and the electrical conductivity. Due to the short mean free path (that is typically the distance of a few lattice parameters), the charge carrier probes the magnetization on a very short length scale. As a result, for these systems a strong interplay between local magnetic order and electric properties exists. Also, there is a large onsite Hund's energy, which for $\mathrm{Mn}^{3+}$ is about $1.5 \mathrm{eV}$. A direct consequence of Hund's interaction is a large spin splitting of the conduction band into majority and minority subbands in the ferromagnetic phase. In optimally doped compounds, e.g., $\mathrm{La}_{0.7} \mathrm{Ca}_{0.3} \mathrm{MnO}_{3}$ and $\mathrm{La}_{0.7} \mathrm{Sr}_{0.3} \mathrm{MnO}_{3}$, the $e_{g}^{\uparrow}(\mathrm{Mn})$ holes are itinerant while $t_{g}^{\downarrow}$ $(\mathrm{Mn})$ electrons are localized, i.e., the ground state electrons are nearly perfectly spin polarized.

Note that in general, the Fermi level $E_{F}$ intersects both up and down bands, so that densities of states of both the $\uparrow$ and $\downarrow$ carriers are present at $E_{F}$. According to the general classification [6], when the $\uparrow$ carriers are itinerant and the $\downarrow$ carriers are localized (or visa versa), we have transport half-metal, classified as a type $\mathrm{III}_{A}$ (or $\mathrm{III}_{B}$ ) half-metal. (The suffix $A$ or $B$ indicates whether the conduction electrons are $\uparrow$ or $\downarrow$ with respect to magnetization direction.) Thus, doped manganites are type III $_{A}$ half-metallic ferromagnets (HMFs). For further discussion, it is noteworthy that numerous experimental results, including transport studies [33-37], indicate that doped manganites are, in fact, "bad" metals even in at low temperature.

Theoretically, HMFs has been justified by band structure calculations [38-43]. For the first time direct experimental evidence of half-metallic density of states in ferro- magnetic $\mathrm{La}_{0.7} \mathrm{Ca}_{0.3} \mathrm{MnO}_{3}$ was obtained by scanning tunneling spectroscopy by Wei et al. [44]. These results were then confirmed by optical [5,45-48] and transport measurements [15,22-29,49-55]. However, it should be taken into account that different experiments yield quite different value of spin polarization (see Table 1). The reasons are obvious: the electron densities of state at the Fermi level $E_{F}$ must be properly weighted and weighting factor depends on the physical process used in measurements. In tunneling experiments, the weighting factors are the appropriate spin-dependent tunneling matrix elements. In PC experiments depending on the character of transport involved, either ballistic or diffusive, the density of states must be weighted either by the Fermi velocity of the electrons or its square, respectively [56,57], and so on.

\subsection{Fabrication and characterization of point contacts}

A PC is simply a contact between two metals, a metal and a semiconductor, or a metal and a superconductor, with a nanoscale radius. PCs may be fabricated in a number of ways $[12,13]$. Traditionally, most often used is the so-called "needle-anvil" configuration when the sample to be studied is one electrode and the other is a metallic tip, which is gently pressed against the sample surface. Typically, the tip has an ending diameter of some tens of micrometers and is easily deformed during the contact. This means that, as a rule, parallel contacts are formed between the sample and the tip. In general, this is not harmful to spectroscopy, unless the sample is highly inhomogeneous on a length scale compared with tip end.

The needle-anvil technique offers several apparent advantages compared to other techniques: it is nondestructive and several measurements can be carried out on the same sample; there are no restrictions on the sample geometry and one can avoid complex fabrication steps, etc. Yet, there are some objective drawbacks such as poor thermal and mechanical stability of the junction. A surface modification due to uncontrolled surface oxides or other chemical reactions on the surface of both the sample and the tip are also possible and should be taken into account, however, the impact of these effects on, e.g., $P_{C}$ is difficult to quantify.

An important characteristic of a PC is its diameter $d$ (it is assumed that the shape of a contact is a circular orifice). Depending on the value of the $d$, different regimes of conduction are possible. The diameter $d$ of a PC with resistance $R_{N}$ can be estimated using the Wexler interpolation formula [58]:

$$
R_{N} \approx \frac{16}{3 \pi} \frac{\rho l}{d^{2}}+\frac{\rho}{d},
$$

where $\rho$ is the resistivity of the crystal, and $l$ stands for the charge carriers mean free path. The mean free path in the metals can be estimated using the well known expression for conductivity: $\sigma=e^{2} N\left(E_{F}\right) D$, where $D=l v_{F} / 3$ is the 
electron diffusive constant, $N\left(E_{F}\right)$ stands for the density of state, and $v_{F}$ is the electron velocity at the Fermi surface. Using for the parameter values available from literature [33-36], one can get the mean free path for carriers in doped manganites $l \sim 100 \AA$. Then, for a contact with typical resistance $R_{N} \sim 100 \Omega$, we find from Eq. (1) the contact diameter $d \sim 100 \AA$. The relation $l \sim d$ means that the contact with $R_{N} \sim 100 \Omega$ borders between the diffusive $(l<<d)$ and ballistic $(l>>d$ ) types of conductivity. Correspondingly, contacts with lower resistance are close to the diffusive type of conduction and contacts with higher resistance are close to the ballistic type of conduction.

For contacts of $\mathrm{N}$ metal with $\mathrm{SC}$ there is an additional characteristic length. The solution of the Bogoliubov-de Gennes equations near an N/SC interface yields that the AR does not occur abruptly at the interface but over a length scale of the order of the superconducting coherence lengths $\xi_{S}$ and $\xi_{N}$ in both directions, respectively. In general, $\xi_{S}$ and $\xi_{N}$ characterize the so-called "proximity" effects. Namely, $\xi_{N}$ is the length over which superconducting correlations spread into $\mathrm{N}$ metal, and $\xi_{S}$ is the length over which superconductivity of SC can be depressed due to contact with $\mathrm{N}$ metal [59]. If the contact size is considerably smaller than $\xi_{S}$ proximity effects can be neglected; if not, proximity effects should be taken into account [13].

\section{Theoretical basis of the AR spectroscopy of current spin polarization}

As was discovered in the early 1960s, at energies below the superconducting gap, a charge transport through a normal nonmagnetic metal being in contact with a SC is possible only due to a specific process called Andreev reflection [8]: a two-particle process in which, in the $\mathrm{N}$ metal, an incident electron above the Fermi energy $E_{F}$ and an electron below $E_{F}$ with an opposite momentum and spin are coupled together and transferred across the interface in the SC side forming a Cooper pair in the condensate. Simultaneously, an evanescent hole appears in the $\mathrm{N}$ metal. The charge doubling at the interface enhances the subgap conductance and this phenomenon has indeed been observed in the case of a perfectly transparent interface. The picture is significantly modified when spin comes into play. For a conventional $s$-wave pairing, a Cooper pair is spin-less $(S=0)$ and spin is not carried through the N/SC interface. Therefore, if the $\mathrm{N}$ metal is an $\mathrm{F}$ and there is an imbalance between spin-up and spin-down populations, the $\mathrm{AR}$ is suppressed and the subgap conductance can be reduced below the normal-state value down to zero as the polarization approaches $100 \%$.

Theoretically, the behavior of normal metal-constriction-superconductor (N-c-SC) systems or N-SC point contacts was analyzed by Zaitsev [60]. This work was expanded on by Blonder et al. [61] who added the possibility of a delta-function scattering potential at the N/SC inter- face. This provides a simple means for including the effect of interfacial scattering, allowing the successful modeling of N-SC point contacts where the transport ranges from the high current density, purely ballistic or Sharvin [62] regime, to the low current density, a tunneling regime. Subsequently de Jong and Beenakker [14] pointed out that for an F-SC contact the spin polarization of the conduction electrons in the $\mathrm{F}$ would affect AR, because not every incident electron from the $\mathrm{F}$ is able to be reflected as a hole to form a Cooper pair that can move into the SC. They argued that (for an ideal $\mathrm{F}-\mathrm{SC}$ contact) this will reduce the AR transmission probability to a factor $\left(1-P_{C}\right)$ where $P_{C}$ is the polarization of the current in the F. Given the widespread interest to understanding the spin-dependent current and related spintronic effects in heterogeneous structures, this led to the development of the PCAR method for determining the degree of current spin polarization in ferromagnetic systems of spintronics interest, and to the development of several different models to interpret the F-SC PC data.

Below we very briefly discuss the conventional models of conductivity for a point SC-F contact: ballistic, diffusive, and a contact with possible spin discrimination of transmission at SC/F interface. (For more details see original reports $[14,27,56,57,61,63,64]$, textbook [12] and recent review [13].)

\subsection{Ballistic-type contacts}

The most simplified but theoretically most selfconsistent is the 1D (i.e., all the involved momenta are normal to the interface) model of a PC with ballistic type of conduction. That is, the charge carriers elastic mean free path $l$ is much larger than the diameter of the contact $d$ and there is no scattering in the contact area. The barrier is represented by a repulsive potential located at the interface with a dimensionless amplitude, $\sim Z \delta(x)$ (here it is supposed that the interface lies in the $y z$-lane); the value $Z=0$ describes a clean interface, and values of $Z>5$ correspond to the tunneling type of conductivity. Note that, if the Fermi velocities of $\mathrm{N}$ and SC metals in a contact are different $\left(v_{F N} \neq v_{F S}\right)$, then the effective parameter $Z \neq 0$ even if there is no scattering of carriers at the interface. It can be assumed that almost all the voltage $V$ is applied directly to the contact itself and, therefore, the metals on both sides of the contact are in equilibrium state and can be described by the Fermi distribution functions.

For the F-SC contact with the ballistic type of conductivity, the contact conductance $G$ can be conveniently represented as the sum of two terms [15]:

$$
G(V)=\left(1-P_{C}\right) G_{N S}(V)+P_{C} G_{P S}(V),
$$

the nonpolarized part $G_{N S}$ (which corresponds to an Andreev contact with nonmagnetic metal) and the completely polarized part $G_{P S}$ (which corresponds to a SC-ferromagnetic metal contact with full polarization of charge 
carriers). The Andreev conductance of the N/SC contact, $G_{N S}$, is given by the well-known expression (the BTK model [61]):

$$
\begin{gathered}
G_{N S}(V)= \\
=G_{N N} \int_{-\infty}^{\infty} d n_{F}(\varepsilon-e V, T) / d V\left[1+A_{N}(\varepsilon, Z)-B_{N}(\varepsilon, Z)\right] d \varepsilon .
\end{gathered}
$$

Here $G_{N N}$ is the normal state conductance (or for the case of $e V>>\Delta$ ); the electron energy $\varepsilon$ is measured from the Fermi level of the superconductor; $n_{F}$ is the Fermi function; the function $A_{N}(\varepsilon, Z)$ gives the probability of the $\mathrm{AR}$ and $B_{N}(\varepsilon, Z)$ corresponds to usual reflection.

The conductance of the completely polarized channel $G_{P S}$ can be given in the form:

$$
\begin{gathered}
G_{P S}(V)= \\
=G_{N F} \int_{-\infty}^{\infty} d n_{F}(\varepsilon-e V, T) / d V\left[1+A_{P}(\varepsilon, Z)-B_{P}(\varepsilon, Z)\right] d \varepsilon
\end{gathered}
$$

where $G_{N F}$ is the conductance of the contact for $e V \gg>\Delta$, the parameter $A_{P}(\varepsilon, Z)$ describes the AR of polarized current, and $B_{P}(\varepsilon, Z)$ describes the usual reflection. If $|\varepsilon|<\Delta$, the AR of completely polarized electrons is impossible and $A_{P}(\varepsilon, Z)=0$. The explicit expressions for the amplitudes $A_{N}(\varepsilon, Z), B_{N}(\varepsilon, Z), A_{P}(\varepsilon, Z)$, and $B_{P}(\varepsilon, Z)$, can be found in $[14,27,56,57,61,63,64]$.

\subsection{Diffusive-type contacts}

For contacts with a normal state resistance $R_{N}<100 \Omega$, the diffusive conductivity approximation is more appropriate. Accordingly, the electron momentum is no longer a good quantum number since $l<<d$ but with only elastic scattering in the contact region (i.e., $d<<l_{\mathrm{el}}$, where $l_{\mathrm{el}}$ is the inelastic-scattering length). This limit usually relates to $\mathrm{N}-\mathrm{SC}$ contacts made by rubbing. Typically, it is assumed also that the superconductor remains in the clean limit (i.e., $\left.l_{S}>\xi_{S}\right)$ but, in addition to the N/SC interface, there is a wide layer of disordered ferromagnetic metal (adjacent to the contact area) where the electron mean free path is much shorter than the contact size.

The total conductance of the contact in the diffusive approximation is given by Eqs. (2)-(4), where now all the amplitudes, $A_{N}(\varepsilon, Z), B_{N}(\varepsilon, Z), A_{P}(\varepsilon, Z)$, and $B_{P}(\varepsilon, Z)$, must be accordingly generalized. We will not write out here the extended expressions referring the reader to reports $[13,18,24,27]$.

If not only the mean free path $l$ is much less than the diameter, but the inelastic-scattering length, as well, $d>>l_{\mathrm{el}}$, we deal with the so-called thermal regime of conductance. There is both elastic and inelastic scattering in the contact region. In this case, Joule heating occurs in the contact region and causes a local increase in temperature. The in- terpretation of data obtained on such contacts is an ambiguous task (see, e.g., discussion in $[12,13]$ ).

\subsection{Contacts with spin-dependent transmission}

In spite of the conventional models success in fitting the experiments, several basic questions naturally arise for the SC-F contacts. For example, how to describe spindependent properties of the SC/F interface? Indeed, if the Fermi velocities for spin up and down electrons are different $\left(v_{F \uparrow} \neq v_{F \uparrow}\right)$, then the effective parameter $Z$, and thus all other transmission coefficients, are also different for the majority- and minority - spin bands.

The minimal model $[65,66]$ which describes transport in the SC-F contacts and accounts for the spin-dependent transmission gives the following expressions for zerotemperature conductance:

$$
\begin{aligned}
G_{S F} & =G_{N N}^{-1} \frac{T_{\uparrow} T_{\downarrow}}{\left(1+r_{\uparrow} r_{\downarrow}\right)^{2}-4 r_{\uparrow} r_{\downarrow}(e V / \Delta)^{2}}, \quad e V \leq \Delta, \quad \text { (5a) } \\
G_{S F} & =G_{N N}^{-1} \frac{T_{\uparrow} T_{\downarrow}+\left(T_{\uparrow}+T_{\downarrow}-T_{\uparrow} T_{\downarrow}\right) \beta(e V / \Delta)}{\left[\left(1-r_{\uparrow} r_{\downarrow}\right)+\left(1+r_{\uparrow} r_{\downarrow}\right) \beta(e V / \Delta)\right]^{2}}, \quad e V \geq \Delta,(5 b)
\end{aligned}
$$

where $T_{\sigma}(\sigma=\uparrow$ or $\downarrow)$ is the effective transmission coefficient for electrons with spin up and down, respectively; $r_{\sigma}=\left(1-T_{\sigma}\right)^{1 / 2}, \beta(e V / \Delta)=\left[1-(\Delta / e V)^{2}\right]^{1 / 2}$. The normal state conductance is read as $\mathrm{G}_{N N}=\left(T_{\uparrow}+T_{\downarrow}\right) / 4$, and the current polarization is defied as $P_{C}=\left(T_{\uparrow}-\mathrm{T}_{\downarrow}\right) /\left(\mathrm{T}_{\uparrow}+\mathrm{T}_{\downarrow}\right)$. The contact conductivity at $T \neq 0$ is given by Eq. (3) with $G_{S F}(5 a)$, (5b) replacing the factor $\left[1+A_{N}(\varepsilon, Z)-B_{N}(\varepsilon, Z)\right]$. The main approximation of this model is the assumption that we can describe the point contact with a single pair of transmission coefficients, $T_{\uparrow}$ and $T_{\downarrow}$, which is finally justified by the agreement with the experiment.

Summarizing, the effects of the interface structure, specific details of the contact preparation method, etc. should also be taken into account when finding the polarization of the ferromagnetic materials. In fact, one needs to test all models to obtain trustful value of spin polarization. For example, if the contact is in the diffusive limit, then fitting the data using the ballistic model may result in an underestimated current polarization. For more detailed discussion of these questions we refer to Refs 12,13 and references therein. The examples of such fitting will be discussed in the next section.

\section{Conventional half-metallic characteristics of point contacts}

We start with the results from samples that reveal conventional properties of sSC-HMF PCs. Conventional scenario predicts that in the extreme case of a completely spin polarized metal being in contact with a singlet $s$-wave $\mathrm{SC}$ ( $\mathrm{SSC}$ ) the AR is suppressed. Thus, a fingerprint of the conventional half-metallic characteristics of $\mathrm{PC}$ is a quite visible increase of the contact's resistivity just after su- 
perconducting transition temperature of the electrode. In Fig. 1, representative low-temperature characteristics of $\mathrm{Pb} / \mathrm{La}_{0.65} \mathrm{Sr}_{0.35} \mathrm{MnO}_{3} \mathrm{PC}$ are shown. The $I-V$ dependence is shown in the main panel, the top inset exhibits the temperature dependence of the contact's resistance $R(T)$, and the bottom inset illustrates the contact's AR spectra, the differential conductance $d I / d V$. The quasiparticles energy gap of $\mathrm{Pb}$ is pointed in the bottom inset by arrows. (Further on, we simply denote the position of $d I / d V$ minimum by $\Delta$. For a PC with not too large $\Gamma$ parameter, introduced by Dynes et al. [67], this value does not differ too much from the true energy gap [68].) Sharp increase of the contact's resistivity just after $T_{C}(\mathrm{~Pb})=7.2 \mathrm{~K}$ is the main distinguishing feature of such type PCs. Below $T_{C}(\mathrm{~Pb})$ we observe excess voltage and almost doubling of the contact's resistivity. Reduction in the conductivity for the PC shown is about $50 \%$.

Similar results for Ca-doped manganite are shown in Fig. 2. Here temperature dependence of the $\mathrm{Pb} / \mathrm{La}_{0.67} \mathrm{Ca}_{0.33} \mathrm{MnO}_{3}$ contact resistance is shown in the main panel. Inset in this figure illustrates the AR spectra for this PC.

Figure 3 illustrates normalized conductivity $(d I / d V) /(I / V)$ of some other contacts: $\mathrm{MgB}_{2} / \mathrm{La}_{0.65} \mathrm{Ca}_{0.35} \mathrm{MnO}_{3}$ (1) $\mathrm{Pb} / \mathrm{La}_{0.7} \mathrm{Sr}_{0.3} \mathrm{MnO}_{3}$ (2), and $\mathrm{Pb} / \mathrm{La}_{0.65} \mathrm{Ca}_{0.35} \mathrm{MnO}_{3}$ (3) (the graphs are shifted along the vertical axis for clarity); note that in case (3) the contact is in the thermal regime. Fitting the model predictions for different junction conduction to the experimental data (in Fig. 3, theory is solid line, expe-

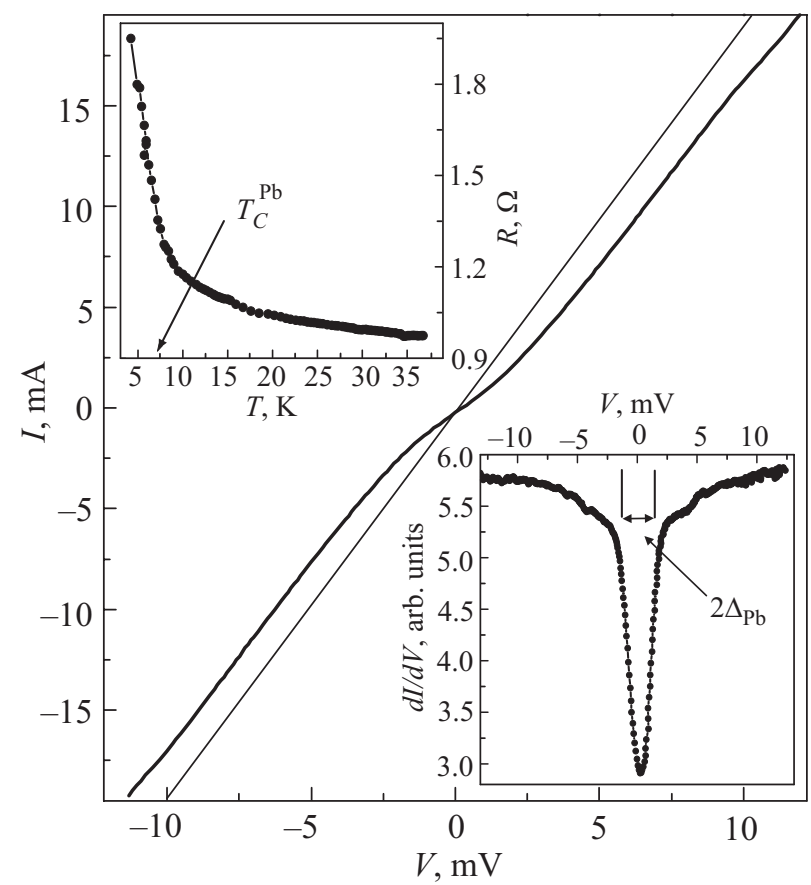

Fig. 1. The current-voltage dependence of the $\mathrm{Pb} / \mathrm{La}_{0.65} \mathrm{Sr}_{0.35} \mathrm{MnO}_{3}$ contact without visible superconducting proximity effect; $T=$ $=4.2 \mathrm{~K}$. Top inset: the temperature dependence of the contact's resistance $R(T)$; arrow indicates $T_{C} \mathrm{~Pb}=7.2 \mathrm{~K}$. Bottom inset: the contact's Andreev-reflection spectra; $T=4.2 \mathrm{~K}$; arrows indicate superconducting gap of $\mathrm{Pb}$. Adopted from Ref. 29.

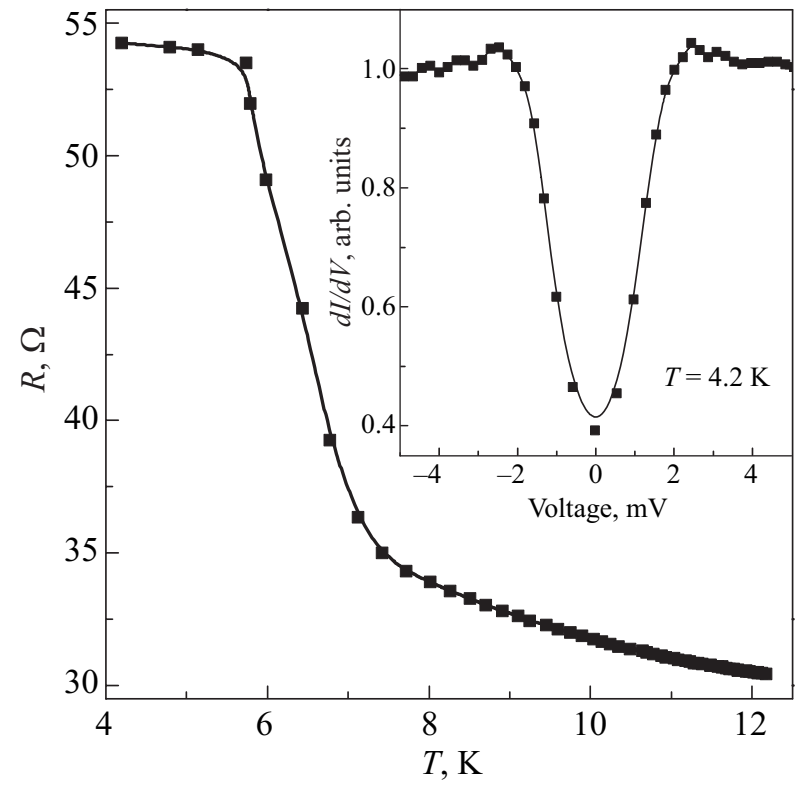

Fig. 2. Temperature dependence of a resistance of $\mathrm{Pb} / \mathrm{La} 0.67 \mathrm{Ca} 0.33 \mathrm{MnO}_{3}$ contact. Inset: Andreev-reflection spectra. Adopted from Ref. 28.

riment is symbols) one can restore the degree of charge carrier spin polarization for a given compound. The degree of spin polarization of charge carriers obtained by this

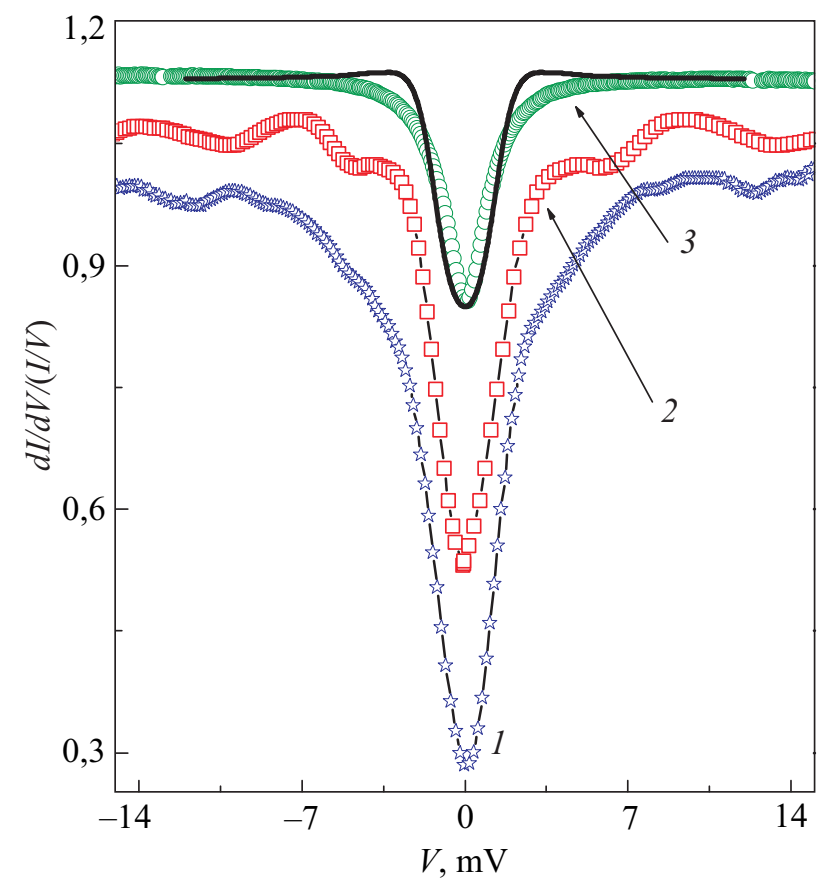

Fig. 3. Normalized conductivity $(d I / d V) /(I / V)$ of $\mathrm{MgB}_{2} / \mathrm{La}_{0.65} \mathrm{Ca}_{0.35} \mathrm{MnO}_{3} \quad$ (1) $\quad \mathrm{Pb}_{2} \mathrm{La}_{0.7} \mathrm{Sr}_{0.3} \mathrm{MnO}_{3} \quad$ (2), and $\mathrm{Pb} / \mathrm{La}_{0.65} \mathrm{Ca}_{0.35} \mathrm{MnO}_{3}$ (3) PCs (the graphs are shifted along the vertical axis for clarity). In case (3), the contact is in the thermal regime (theory is solid line, experiment is symbols). The degree of the spin polarization of charge carriers obtained by fitting are $P_{C} \approx 83,78$, and $65 \%$, respectively. $T=4.2 \mathrm{~K}$. Adopted from Ref. 27. 
Table 1. Degree of spin polarization deduced for doped manganites. Technique: SPT — spin-polarized tunneling; SPPh — spinpolarized photoemission; $\mathrm{BC}$ - band calculation; PCAR — pointcontact Andreev reflection; STS — scanning tunneling spectroscopy; ARPES - angle-resolved photoemission spectroscopy

\begin{tabular}{c|c|c}
\hline \hline $\mathrm{Compound}$ & Spin polarization & Technique, Refs. \\
\hline \hline $\mathrm{La}_{0.67} \mathrm{Sr}_{0.33} \mathrm{MnO}_{3}$ & $36 \%$ & $\mathrm{BC},[38]$ \\
$\mathrm{La}_{0.67} \mathrm{Sr}_{0.33} \mathrm{MnO}_{3}$ & $72 \%$ & $\mathrm{SPT},[53]$ \\
$\mathrm{La}_{0.67} \mathrm{Sr}_{0.33} \mathrm{MnO}_{3}$ & $54 \%$ & $\mathrm{SPT},[51]$ \\
$\mathrm{La}_{0.67} \mathrm{Sr}_{0.33} \mathrm{MnO}_{3}$ & $81 \%$ & $\mathrm{SPT},[52]$ \\
$\mathrm{La}_{0.7} \mathrm{Sr}_{0.3} \mathrm{MnO}_{3}$ & $100 \%$ & $\mathrm{SPPh},[5]$ \\
$\mathrm{La}_{0.7} \mathrm{Sr}_{0.3} \mathrm{MnO}_{3}$ & $78 \%$ & PCAR, [27] \\
$\mathrm{La}_{0.7} \mathrm{Sr}_{0.3} \mathrm{MnO}_{3}$ & $58 \%-92 \%$ & PCAR, [22] \\
$\mathrm{La}_{0.7} \mathrm{Sr}_{0.3} \mathrm{MnO}_{3}$ & $78 \%$ & PCAR, [23] \\
$\mathrm{La}_{0.6} \mathrm{Sr}_{0.4} \mathrm{MnO}_{3}$ & $83 \%$ & PCAR, [23] \\
$\mathrm{La}_{0.7} \mathrm{Sr}_{0.3} \mathrm{MnO}_{3}$ & $78 \%$ & PCAR, [15] \\
$\mathrm{La}_{0.66} \mathrm{Sr}_{0.34} \mathrm{MnO}_{3}$ & $100 \%$ & BC, [42] \\
$\mathrm{La}_{0.6} \mathrm{Sr}_{0.4} \mathrm{MnO}_{3}$ & $100 \%$ & ARPE, [48] \\
$\mathrm{La}_{2 / 3} \mathrm{Sr}_{1 / 3} \mathrm{MnO}_{3}$ & $90 \%$ & $\mathrm{SPT},[55]$ \\
$\mathrm{La}_{2 / 3} \mathrm{Sr}_{1 / 3} \mathrm{MnO}_{3}$ & $78 \%-82 \%$ & PCAR, [24] \\
$\mathrm{La}_{0.7} \mathrm{Sr}_{0.3} \mathrm{MnO}_{3}$ & $100 \%$ & $\mathrm{SPPh},[46]$ \\
$\mathrm{La}_{0.7} \mathrm{Sr}_{0.3} \mathrm{MnO}_{3}$ & $83 \%$ & PCAR, [23] \\
$\mathrm{La}_{1-x} \mathrm{Ca}_{x} \mathrm{MnO}_{3}$ & $100 \%$ & BC, [39] \\
$\mathrm{La}_{0.65} \mathrm{Ca}_{0.35} \mathrm{MnO}_{3}$ & $65 \%-83 \%$ & PCAR, [27] \\
$\mathrm{La}_{2 / 3} \mathrm{Ca}_{1 / 3} \mathrm{MnO}_{3}$ & $36 \%$ & BC, [38] \\
$\mathrm{La}_{0.7} \mathrm{Ca}_{0.3} \mathrm{MnO}_{3}$ & $100 \%$ & $\mathrm{STS},[44]$ \\
$\mathrm{La}_{1-x} \mathrm{Ca}_{x} \mathrm{MnO}_{3}$ & $80 \%$ & BC, [43] \\
$\mathrm{La}_{0.75} \mathrm{Ca}_{0.25} \mathrm{MnO}_{3}$ & $100 \%$ & BC, [40] \\
$\mathrm{La}_{0.7} \mathrm{Ca}_{0.3} \mathrm{MnO}_{3}$ & $86 \%$ & $\mathrm{SPT},[54]$ \\
$\mathrm{La}_{0.7} \mathrm{Ce}_{0.3} \mathrm{MnO}_{3}$ & $100 \%$ & $\mathrm{SPPh},[47]$ \\
$\mathrm{La}_{0.7} \mathrm{Ce}_{0.3} \mathrm{MnO}_{3}$ & $100 \%$ & BC, [41] \\
\hline \hline & &
\end{tabular}

mode for the PCs shown in Fig. 3 is $P_{C}$ equals 83, 78, and $65 \%$, respectively.

The values of charge carrier spin polarization obtained for doped manganites are summarized in Table 1. In spite of some scattering in absolute magnitudes, in general, the data in the Table yields that the degree of spin polarization of the current can be unambiguously determined using the PCAR method.

As already mentioned, the electron and the hole involved in the AR are coherently coupled. The phase-coherent electron-hole conversion results in a nonzero pair amplitude in the $\mathrm{F}$ metal. For singlet pairing in the dirty limit, superconducting coherence length is $\xi_{F} \sim\left(D_{F} / 2 \pi T_{\text {Curie }}\right)^{1 / 2}$ and for manganites one obtains an extremely short distance $\xi_{F} \sim 5-7 \AA$. (Here $D_{F}$ is the diffusivity of the F metal; we choose $\hbar=k_{B}=1$.) Contribution of such a small region to the contact's resistance is less than $1 \%$. That is indeed observed for the most part of the samples [15,22-29,4955]. However, for some of the cases, the measured contact's spectra revealed very distinct features which were interpreted as manifestation of an unconventional proximity effect.

\section{Proximity affected contacts, “anomalous" Andreev reflection}

Typically, the normal state resistivity of proximity affected contacts is larger than that for the contacts without proximity effect (PE). Following the conventional physics [see discussion below Eq. (1)], the conductivity of these contacts should be close to the ballistic type and, accordingly, the spectroscopic characteristics would be more perfect than those shown in Figs. 1-3. Yet, the characteristics observed were fundamentally different.

Figure 4 illustrates representative low-temperature characteristics of $\mathrm{Pb} / \mathrm{La}_{0.7} \mathrm{Sr}_{0.3} \mathrm{MnO}_{3}$ proximity affected $\mathrm{PC}$. As in Fig. 1, the $I-V$ dependence is shown in the main panel, the top inset exhibits the temperature dependence of the contact's resistance $R(T)$, and the bottom inset illustrates the contact's AR spectra. In contrast to the data in Fig. 1, quite visible decrease of the contact's resistivity is seen just after superconducting transition of a SC tip. Decrease of the PC resistivity is the main distinguishing feature of proximity affected contacts. Also, in contrast to the data in Fig. 1, below $T_{C}(\mathrm{~Pb})=7.2 \mathrm{~K}$ we now observe excess current and almost doubling of the contact's conductivity.

Figure 5 illustrates the properties of another $\mathrm{Pb} / \mathrm{La}_{0.7} \mathrm{Sr}_{0.3} \mathrm{MnO}_{3} \quad \mathrm{PC}$. In comparison with the $\mathrm{PC}$ in Fig. 4, the normal-state resistivity of this contact is about six times larger; however, it demonstrates all specific features of a proximity affected contact, namely: excess current, anomalous AR spectra, proximity induced gap, $\Delta_{\text {tr }}$,

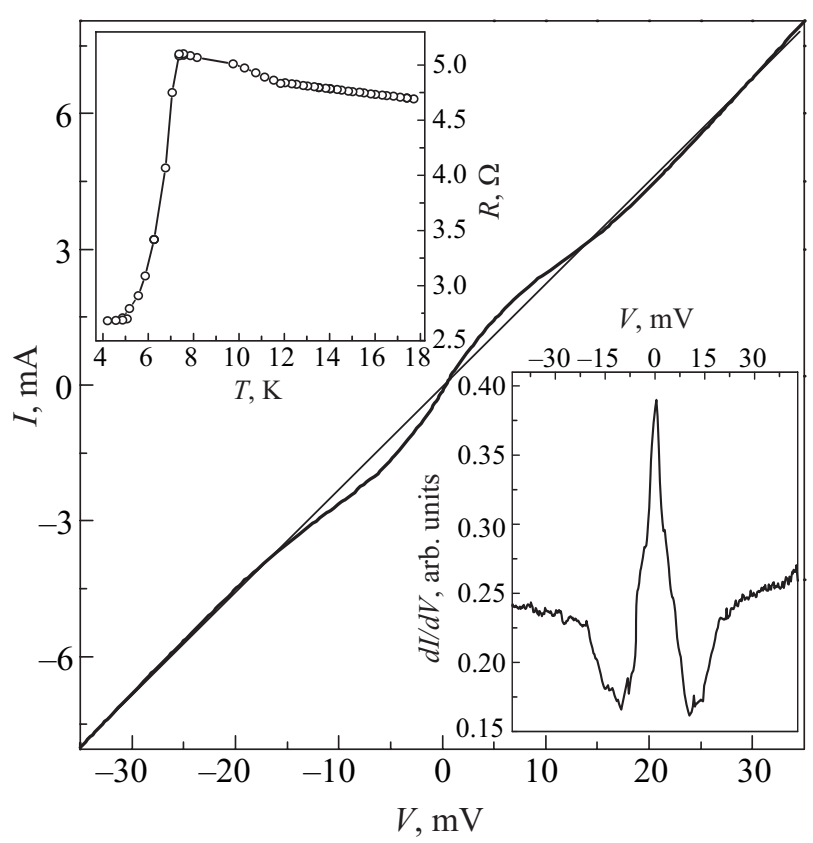

Fig. 4. The current-voltage dependence of the proximity affected $\mathrm{Pb} / \mathrm{La}_{0.7} \mathrm{Sr}_{0.3} \mathrm{MnO}_{3}$ contact; $T=4.2 \mathrm{~K}$. Top inset: the temperature dependence of the contact's resistance $R(T)$. Bottom inset: the contact's Andreev-reflection spectra at $T=4.2 \mathrm{~K}$. Reproduced form Ref. 29. 


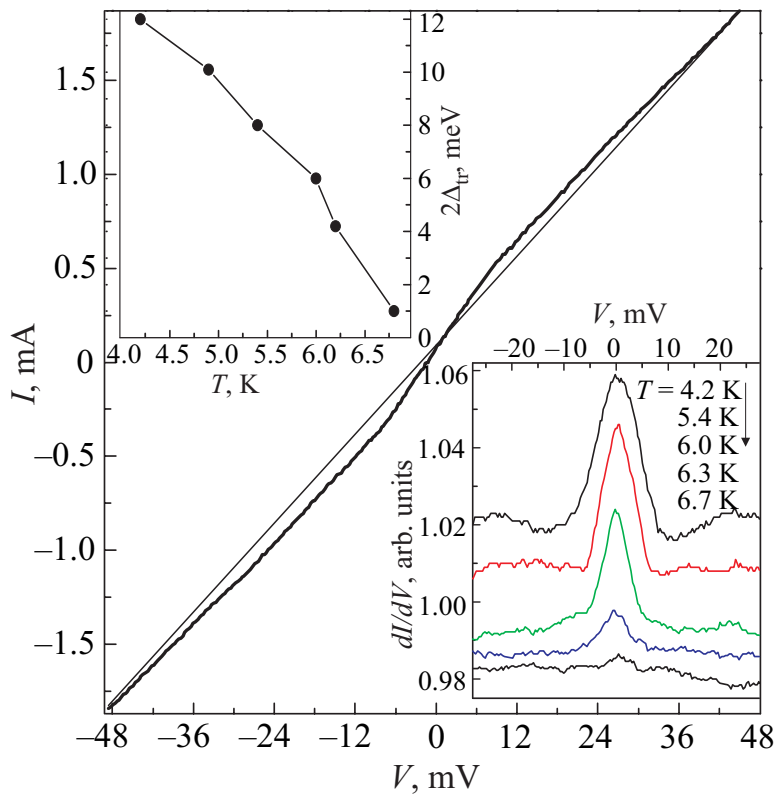

Fig. 5. The current-voltage dependence of the proximity affected $\mathrm{Pb} / \mathrm{La}_{0.7} \mathrm{Sr}_{0.3} \mathrm{MnO}_{3}$ contact; $T=4.2 \mathrm{~K}$. Top inset: the evolution of the proximity induced quasiparticle gap $\Delta_{\text {tr }}$ with temperature. Bottom inset: the temperature dependence of the contact's Andreev-reflection spectra. The curves are shifted for clarity. Reproduced form Ref. 29.

which is much larger than that for $\mathrm{Pb}$. In the bottom inset, the evolution of the AR spectra with temperature is shown. These data directly prove that the anomalous behavior of the junction is due to the superconducting state of $\mathrm{Pb}$. In the top inset, the temperature dependence of proximity induced quasiparticle gap is shown.

In Figs. 6 and 7, the normalized conductance spectra for some additional proximity affected $\mathrm{PCs}$ of $\mathrm{Pb}$ or $\mathrm{MgB}_{2}$ with films of $\mathrm{Ca}$ - and $\mathrm{Sr}$-doped manganites are shown. For low voltage, the fine structure of the AR spectra is directly visible. This structure is the so-called subharmonic gap structure (SGS) and manifests itself in a set of downward peaks in the differential conductance that are pointed by labeled arrows in Figs. 6 and 7.

According to contemporary models (see, for example, Refs. 69-71 and references therein), for SC-N-SC weak links or short constrictions SC-c-SC between two superconductors, the differential conductance $d I / d V$ drops fairly abruptly due to multiple ARs. These conductance drops appear at voltage that correlates with the energy of quasiparticle gaps divided by integers. The voltages at which the conductance SGS appears (roughly, because the boundary conditions at interface are also important [71]) are: $\mathrm{eV}=$ $=\Delta_{1} / n, e V_{m}=\Delta_{2} / m$, and $e V_{l}=\left(\Delta_{1}+\Delta_{2}\right) /(2 l+1)$, where the integers $(l, n, m)$ are restricted depending on the energy gap ratio $\left(\Delta_{1} / \Delta_{2}\right)$. What is important for us here is that the resonances can be observed only if both electrodes are superconductors. So, the observation of the SGS (Figs. 6

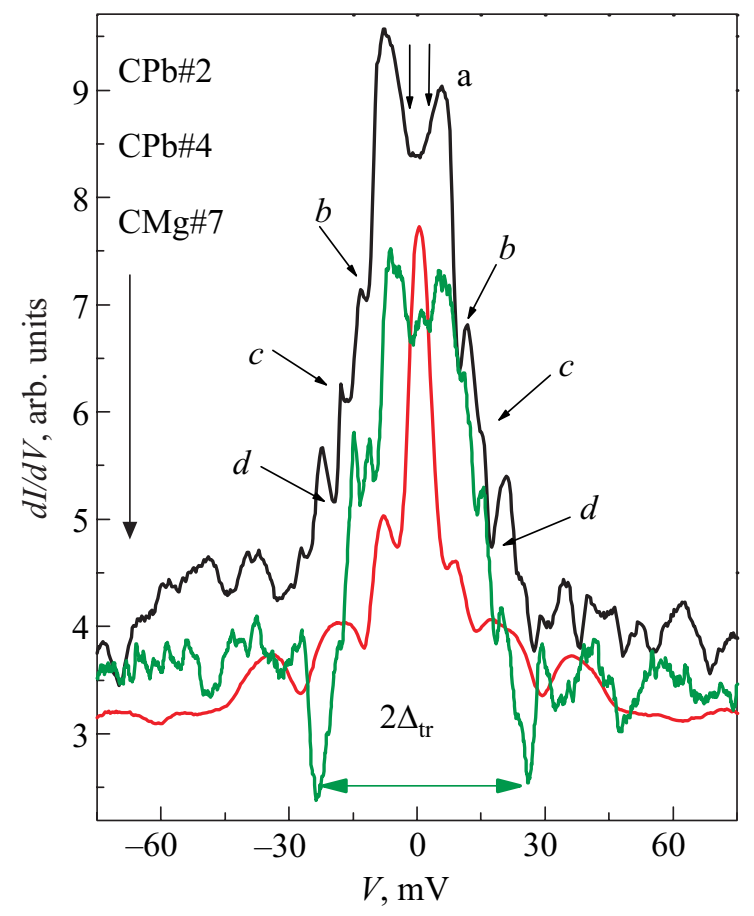

Fig. 6. Normalized conductance spectra for proximity affected $\mathrm{Pb} / \mathrm{La}_{0.65} \mathrm{Ca}_{0.35} \mathrm{MnO}_{3}$ point contacts ( $\mathrm{CP} \# 2$ and $\mathrm{CP} \# 4$ ), and $\mathrm{MgB}_{2} / \mathrm{La}_{0.65} \mathrm{Ca}_{0.35} \mathrm{MnO}_{3}$ contact $(\mathrm{CMg} \# 7)$. The curves are shifted for clarity. The arrows indicate the energies of the subharmonic gap resonances for $\mathrm{CP} \# 2$ contact. See Table 3 for classification of the resonances; $\Delta_{\mathrm{tr}}$ is the apparent proximity induced singleparticle gap of the $\mathrm{La}_{0.65} \mathrm{Ca}_{0.35} \mathrm{MnO}_{3}$. Reproduced form Ref. 28 .

and 7) is a strong argument in favor of the fact that the manganites are in a superconducting state with actual gap independent on SC partner.

From the experimental results in Figs. 4-7, we extract that the proximity induced single-particle gap at the $\mathrm{Pb} / \mathrm{La}_{0.7} \mathrm{Sr}_{0.3} \mathrm{MnO}_{3}$ and $\mathrm{Pb} / \mathrm{La}_{0 .}{ }_{65} \mathrm{Ca}_{0 .}{ }_{35} \mathrm{MnO}_{3}$ interface is as large as $\Delta_{\mathrm{tr}} \approx 18-20 \mathrm{meV}$. Note that the detected gap $\Delta_{\mathrm{tr}}$ is much larger than that of $\mathrm{Pb}$ or of $\mathrm{MgB}_{2}$; for these $\mathrm{SCs}$ we have at $T=4.2 \mathrm{~K}: \Delta=1.41 \mathrm{meV}$ for $\mathrm{Pb}$, and for $\mathrm{MgB}_{2}$ two superconducting energy gaps (the $\sigma$ and $\pi$ gaps) with $\Delta_{\pi}=2.3 \mathrm{meV}$ and $\Delta_{\sigma}=7.1 \mathrm{meV}$ for $\mathrm{MgB}_{2}$ [72]. Knowing energy gap for the SC tip, the subharmonic gap structure can be classified as shown in Table 2 .

To conclude on this section, we summarize the main results detected for proximity affected PCs (Figs. 4-7). Firstly, such principal fact as spectacular drop of the contact's resistance with the onset of the SC tip superconductivity has been observed. Secondly, the subharmonic gap resonances due to multiple AR are directly visible. Thirdly, in proximity affected $\mathrm{PCs}$, the magnitude of a proximity induced gap is much larger than that of the SC tip and may be as large as $\Delta_{\text {tr }} \approx 18-20 \mathrm{meV}$. These facts strongly suggest that both electrodes are in a superconducting state with independent gaps. All these anomalies are observed only in the superconducting state of the tip. 


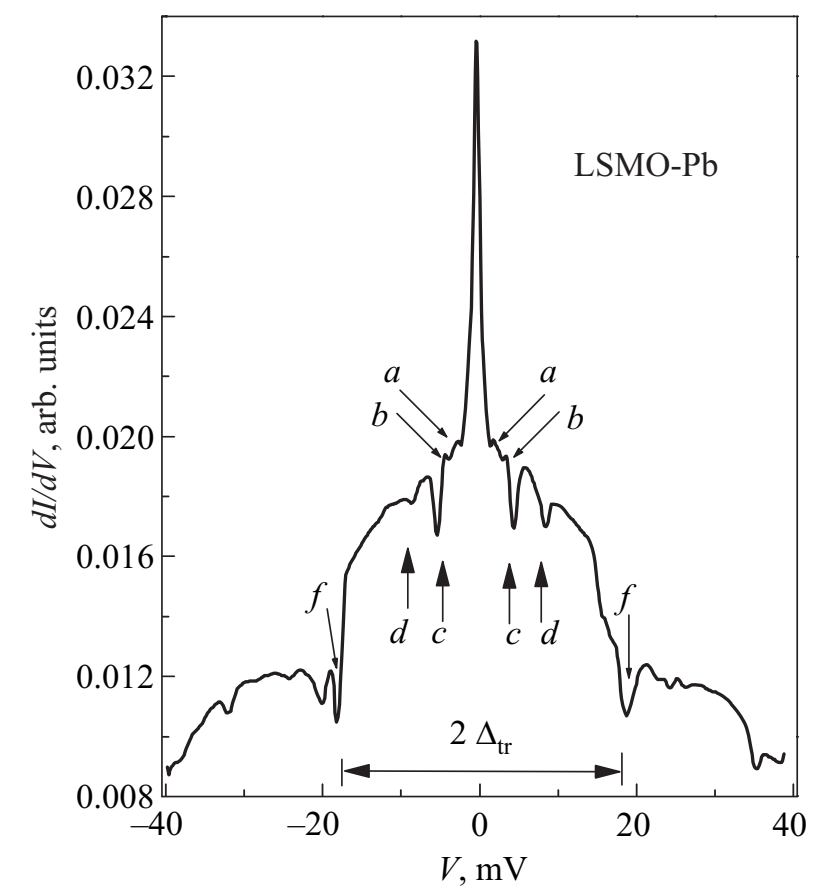

Fig. 7. Those as in Fig. 6 for proximity affected $\mathrm{Pb} / \mathrm{La}_{0.7} \mathrm{Sr}_{0.3} \mathrm{MnO}_{3}$ point contact at $4.2 \mathrm{~K}$. Reproduced form Ref. 29.

To proceed further, let us briefly discuss models extending the conventional concepts of interplay between superconductivity and ferromagnetism.

Table 2. The voltage corresponding to the SGS in Figs. 6 and 7, point contacts $\mathrm{Pb}-(\mathrm{La}, \mathrm{Ca}) \mathrm{MnO}_{3}$ and $\mathrm{Pb}-(\mathrm{La}, \mathrm{Sr}) \mathrm{MnO}_{3}$, respectively; here $\Delta_{\mathrm{Pb}} \approx 1.4 \mathrm{meV}, \Delta_{\mathrm{tr}} \approx \Delta_{L C M O} \approx \Delta_{L S M O} \approx 18-20 \mathrm{meV}$

\begin{tabular}{c|c}
\hline \hline Label (Fig. 6, CPb\#2) & Voltage \\
\hline \hline$a$ & $\Delta_{\mathrm{Pb}}$ \\
$b$ & $\Delta_{\mathrm{LCMO}} / 2$ \\
$c$ & $2 \Delta_{\mathrm{LCMO}} / 3$ \\
$d$ & $\Delta_{\mathrm{LCMO}}$ \\
\hline \hline Label (Fig. 7) & Voltage \\
\hline$a$ & $\Delta_{\mathrm{Pb}}$ \\
$b$ & $\left(\Delta_{\mathrm{Pb}}+\Delta_{\mathrm{LSMO}}\right) / 5$ \\
$c$ & $\Delta_{\mathrm{LSMO}} / 3$ \\
$d$ & $\Delta_{\mathrm{LSMO}} / 2$ \\
$f$ & $\Delta_{\mathrm{LSMO}}$ \\
\hline \hline
\end{tabular}

\section{Triplet transport in proximity affected SSC-HMF point contacts}

Conventional model of PE predicts that proximity induced superconductivity decays rapidly (a few nanometers) inside the $\mathrm{F}$ layer owing to the incompatible nature of singlet superconductivity and ferromagnetic order, and thus proximity induced superconductivity of the F metal can be neglected [59]. This expectation was indeed confirmed in various materials and geometries. On the other hand, an increasing number of experimental facts [73-82] present clear evidences that a simple physical interpretation of the PE, reading that the Cooper pairs are broken by strong exchange field in the F layer, is in reality too simplistic, and an extension of the existing concepts of interplay between superconductivity and ferromagnetism is needed. Long-ranged PE has been observed in a variety of ferromagnetic materials, including wires [74,74], bi- and multilayers [75,81], half metallic $\mathrm{CrO}_{2}[78]$ rare earth metals with helical magnetic structure [77], etc.

Concerning the manganites, note that much earlier Kasai et al., investigated current-voltage characteristics of $\mathrm{YBCO} / \mathrm{La}_{1-x} \mathrm{Ca}_{x} \mathrm{MnO}_{z} / \mathrm{YBCO}$ [83] and $\mathrm{YBCO} /$ $\mathrm{La}_{1-x} \mathrm{Sr}_{x} \mathrm{MnO}_{z} / \mathrm{YBCO}$ [84] layered junctions (here $\mathrm{YBCO}$ stands for $\mathrm{YBa}_{2} \mathrm{Cu}_{3} \mathrm{O}_{y}$ ). Surprisingly, supercurrent was observed through magnetic barrier as thick as $300 \mathrm{~nm}$ for junctions with $\mathrm{La}_{1-x} \mathrm{Ca}_{x} \mathrm{MnO}_{z}$ and $200 \mathrm{~nm}$ for junctions with $\mathrm{La}_{1-x} \mathrm{Ca}_{x} \mathrm{MnO}_{z}$. That is for barrier's thicknesses much larger than a distance one may expect based on conventional proximity effect. Yet, this phenomenon occurred only for manganese oxides with $x=0.3-0.4$. The authors suggested the results may be due to a novel proximity effect between YBCO and doped manganites. Further neutron measurements on $\mathrm{YPrBaCuO} / \mathrm{La}_{0} .7 \mathrm{Ca}_{0.3} \mathrm{MnO}_{3}$ multilayers [85] suggest a possibility of inducing spin-triplet superconducting phase in manganite layers, which could be the source of long-range proximity effect observed in $\mathrm{La}_{0.7} \mathrm{Ca}_{0.3} \mathrm{MnO}_{3} / \mathrm{YBCO}$ multilayers $[76,86]$, trilayers [87], and bilayers [89].

From the theoretical viewpoint, a hybrid system of an $\mathrm{F}$ with a uniform exchange field in a metallic contact with a SC is well understood and the PE may be described by taking into account the splitting of electronic bands of opposite spins [59]. The situation becomes more complicated if the magnetic structure is inhomogeneous. Theories [8993] predict the appearance of the long-range unconventional PE if there is spatial variation of magnetization (or exchange field) at the $\mathrm{SC} / \mathrm{F}$ interface. Particularly, the triplet components of correlations need to be taken into consideration with a characteristic coherence length of $\xi_{F}=\left(D_{F} / 2 \pi T\right)^{1 / 2}$ that can be as large as $\sim 100 \mathrm{~nm}$ at low temperatures.

Before giving a qualitative explanation of the results detected on PE PCs (Figs. 4-7), we summarize the physics of proximity effect at spin-active $\mathrm{SSC} / \mathrm{F}$ interface which looks as follows (for details see, e.g., $[92,93]$ and references therein).

\subsection{Triplet pairing in $s S C / F$ heterostructures}

As it is well known, superconducting correlations are quantified by the anomalous Green's function (see, e.g., [94]): $\mathrm{G}_{\alpha \beta}\left(\mathbf{r}_{1}, \tau_{1} ; \mathbf{r}_{2}, \tau_{2}\right)=\left\langle T_{\tau} \Psi_{\alpha}\left(\mathbf{r}_{1}, \tau_{1}\right) \Psi_{\beta}\left(\mathbf{r}_{2}, \tau_{2}\right)>\right.$. Here all notations are conventional: the field operators $\Psi_{\alpha}\left(\mathbf{r}_{\mathrm{i}}, \tau_{\mathrm{i}}\right)$ hold anticommutation relations, $\alpha$ and $\beta$ are spin indexes, $T_{\tau}$ is an ordering operator for imaginary time $\tau$, etc. The 
Pauli principle requires that this function changes sign when the two particles are interchanged. For Fourier transformed Green's function its reads:

$$
\mathrm{G}_{\alpha \beta}\left(\mathbf{p}, \omega_{n}\right)=-\mathrm{G}_{\beta \alpha}\left(-\mathbf{p},-\omega_{n}\right) .
$$

The symmetry restriction on Eq. (6) in spin, $S$, momentum, $\mathbf{p}$, and Matsubara frequency, $\omega_{n}=(2 n+1) \pi T$, can be satisfied in four different ways (see, e.g., Table 1 in Ref. 92).

It is convenient to separate the Green's function $\mathrm{G}_{\alpha \beta}\left(\mathbf{p}, \omega_{n}\right)$ into singlet and triplet components as

$$
\mathrm{G}_{\alpha \beta}\left(\mathbf{p}, \omega_{n}\right)=\mathrm{G}_{\mathrm{s}}\left(\mathbf{p}, \omega_{n}\right)\left(\mathrm{i} \sigma_{y}\right)_{\alpha \beta}+\mathrm{G}_{\mathrm{tr}}\left(\mathbf{p}, \omega_{n}\right)\left(\sigma \mathrm{i} \sigma_{y}\right)_{\alpha \beta} .
$$

Here $\sigma$ is the vector of the three Pauli matrices. The singlet spin matrix (i $\left.\sigma_{y}\right)_{\alpha \beta}$ is odd under the interchange $\alpha \leftrightarrow \beta$, while the three triplet matrices $\left(\sigma i \sigma_{y}\right)_{\alpha \beta}$ are even.

To illustrate the physics, we consider a case of a superconductor-weak ferromagnet heterostructure in the ballistic transport regime and spin-active interface scattering. For simplicity, it was also suggested that the proximity induced pairing amplitudes in the $\mathrm{F}$ are small. In this case, within quasi-classical approximation, the anomalous Green's function follows the (linearized) Eilenberger equations (we follow Ref. 92):

$$
\begin{gathered}
\left(\mathbf{v}_{F} \nabla+2 \omega_{n}\right) f_{\uparrow \downarrow}^{S}=2 \pi \Delta \operatorname{sgn}\left(\omega_{n}\right)-2 i H_{\mathrm{exc}} f_{\uparrow \downarrow}^{\mathrm{tr}} \\
\left(\mathbf{v}_{F} \nabla+2 \omega_{n}\right) f_{\uparrow \downarrow}^{\mathrm{tr}}=-2 i H_{\mathrm{exc}} f_{\uparrow \downarrow}^{s} \\
\left(\mathbf{v}_{F} \nabla+2 \omega_{n}\right) f_{\uparrow \uparrow, \downarrow \downarrow}^{\mathrm{tr}}=0 .
\end{gathered}
$$

$f_{\uparrow \downarrow}^{\mathrm{tr}}=(|\uparrow \downarrow>+| \downarrow \uparrow>) \quad(m=0)$ and $f_{\uparrow \uparrow, \downarrow \downarrow}^{\mathrm{tr}}=(|\uparrow \uparrow>,| \downarrow \downarrow>)$ $(m=1)$ are normalized triplet pairing amplitudes, and $f_{\uparrow \downarrow}^{S}=(|\uparrow \downarrow>-| \downarrow \uparrow>)$ stands for the singlet one. The superconducting gap $\Delta$ is nonzero in the SC, while the exchange field $H_{\text {exc }}$ exists in the F.

Note, the exchange field $H_{\text {exc }}$ is presented only in the equations for the pairing correlations involving two spin bands, $f_{\uparrow \downarrow}^{s}$ and $f_{\uparrow \downarrow}^{\mathrm{tr}}$, and is absent for the pairing correlations involving one spin band $f_{\uparrow \uparrow, \downarrow \downarrow}^{\mathrm{tr}}$. The eigenvalues of the $f_{\uparrow \downarrow}^{S}$ and $f_{\uparrow \downarrow}^{\text {tr }}$ amplitudes for a given $v_{F}$ are $k_{n}^{ \pm}=2\left(\left|\omega_{n}\right| \pm \mathrm{i} H_{\text {exc }}\right) / v_{F}$. Thus, both the singlet $f_{\uparrow \downarrow}^{S}$ and the triplet $f_{\uparrow \downarrow}^{\text {tr }}$ amplitudes oscillate on the clean-limit magnetic length scale $\xi_{F}=v_{F} / 2 H_{\text {exc }}$, and decay exponentially on the length scale $\xi_{n}=v_{F} / 2\left|\omega_{n}\right|$; the latter is dominated by the lowest Matsubara frequency, $\omega_{0}=\pi T$, and occurs on the thermal length scale $\xi_{T}=v_{F} / 2 \pi T$. The equations for the $f_{\uparrow \uparrow, \downarrow \downarrow}^{\text {tr }}$ triplet pairing amplitudes do not contain the exchange field, and these components are monotonic decaying functions on the thermal length scale $\xi_{T}=v_{F} / 2 \pi T$.

Yet, the presence of the $\left.f_{\uparrow \uparrow, \downarrow \downarrow}^{\mathrm{tr}}=(|\uparrow \uparrow>,| \downarrow \downarrow\rangle\right)$ components requires spin-active interface scattering, i.e., appropriate boundary conditions. Indeed, the conversion process between the singlet and equal-spin triplet supercurrents is governed by two important phenomena taking place at the SC/F interface: (i) a spin mixing and (ii) a spin-flip scattering. Spin mixing is the result of scattering phase difference $\theta$ that electrons with opposite spin acquire when scattered (reflected or transmitted) from an interface [95]:

$$
\begin{aligned}
& (|\uparrow \downarrow>-| \downarrow \uparrow>)=>\left(\left|\uparrow \downarrow>\mathrm{e}^{\mathrm{i} \theta}-\right| \downarrow \uparrow>\mathrm{e}^{-\mathrm{i} \theta}\right)= \\
= & (|\uparrow \downarrow>-| \downarrow \uparrow>) \cos (\theta)+i(|\uparrow \downarrow>+| \downarrow \uparrow>) \sin (\theta) .
\end{aligned}
$$

It results from difference in orientations between magnetization of the F film and the spin of quasiparticle, or differences in the wave-vector mismatches for spin up and spin down quasiparticles, etc. It is a robust and ubiquitous feature for interfaces involving spin-polarized ferromagnets. However, triplet spin state $(|\uparrow \downarrow\rangle+\mid \downarrow \uparrow>)$ can transform into equal-spin pair states $(|\uparrow \uparrow>,| \downarrow \downarrow>)$ only if there are spin-flip processes at the interfaces or if quantization direction changes, or both. Its origin depends on microscopic magnetic state at the $\mathrm{SC} / \mathrm{F}$ interface, character of local magnetic moments coupling with itinerant electrons, etc., and even varies from sample to sample. But, the exact microscopic origin of spin-flip processes at the interface is important only for the effective interface scattering matrix $[92,93,96,97]$ and not for superconducting phenomena, since Cooper pairs are of the size of the coherence length $\xi_{S}$ which is much larger than the atomic scale.

Thus, due to spin mixing at the interfaces, a spin triplet $(S=1, m=0)$ amplitude, $f_{\uparrow \downarrow}^{\mathrm{tr}}$, is created and extends from the interface about the length $\xi_{T}=v_{F} / 2 \pi T$ into the $\mathrm{F}$ layer oscillating on very short length scale $\xi_{F}=v_{F} / 2 H_{\text {exc }}$ (in typical cases exchange field $H_{\text {exc }}$ is much larger than $T_{C}$ ). At the same time, triplet pairing correlations with equal spin pairs $(S=1, m=+1$ or $m=-1)$ are also induced (due to spin-flip processes) in the F layer smoothly decaying on the length scale $\xi_{T}=v_{F} / 2 \pi T$. It is worthy to emphasize that it is only the $m=0$ triplet component that is coupled via the spin-active boundary condition to the equal-spin $m=1$ pairing amplitudes in the half-metal. The singlet component in the $s$-wave superconductor, $f_{\uparrow \downarrow}^{S}$, being invariant under rotations around any quantization axis, is not directly involved in the creation of the triplet $m=1$ pairing amplitudes in the half-metal.

Considering the simplest spin-active model in which the scattering matrix is independent on the momentum parallel to the interface, for small constant transmission and small spin-mixing one can obtain analytical expressions for the amplitudes. Referring the reader for details to Ref. 92, for the singlet and triplet amplitudes with zero spin projection we have in the region $\xi_{F}<<x<<\xi_{T}$ :

$$
\begin{gathered}
f_{\uparrow \downarrow}^{S}(l)= \\
=f_{0}\left[-\cos \theta \frac{\sin \left(x / \xi_{F}\right)}{x / \xi_{F}}+\cos \alpha \frac{\cos \left(x / \xi_{F}\right)}{x / \xi_{F}}\right] \exp \left(-x / \xi_{T}\right),
\end{gathered}
$$




$$
\begin{gathered}
f_{\uparrow \downarrow}^{\operatorname{tr}}(l)= \\
=i f_{0}\left[-\cos \theta \frac{\cos \left(x / \xi_{F}\right)}{x / \xi_{F}}-\cos \alpha \sin \theta \frac{\sin \left(x / \xi_{F}\right)}{x / \xi_{F}}\right] \exp \left(-x / \xi_{T}\right) .
\end{gathered}
$$

Here $l$ is Legendre polynomials index; $\alpha$ is an angle between a different spin-quantization axis and $\theta$ stands for the spin-mixing angle of spin-active interface; the amplitude $f_{0}$ is determined by the related matrix elements [92]. The triplet amplitudes with nonzero spin projection in this region is approximately constant and the asymptotic behavior is reached if $x>\xi_{T}$, and is of the form

$$
f_{\uparrow \uparrow, \downarrow \downarrow}^{\mathrm{tr}}(l) \sim i f_{0} \sin \theta \frac{\xi_{T}}{x} \exp \left(-x / \xi_{T}\right) .
$$

In Fig. 8 the first partial wave $(l=0)$ of the singlet $f_{\uparrow \downarrow}^{S}(x)$ and triplet, $f_{\uparrow \downarrow}^{\mathrm{tr}}(x)$ and $f_{\uparrow \uparrow \downarrow \downarrow}^{\mathrm{tr}}(x)$, pairing amplitudes $\left[f_{s}(l=0), f_{t z}(l=0)\right.$ and $f_{t \perp}(l=0)$, respectively] spatial behaviors are shown in clean $\mathrm{sSC} / \mathrm{F}$ structures at tempera-
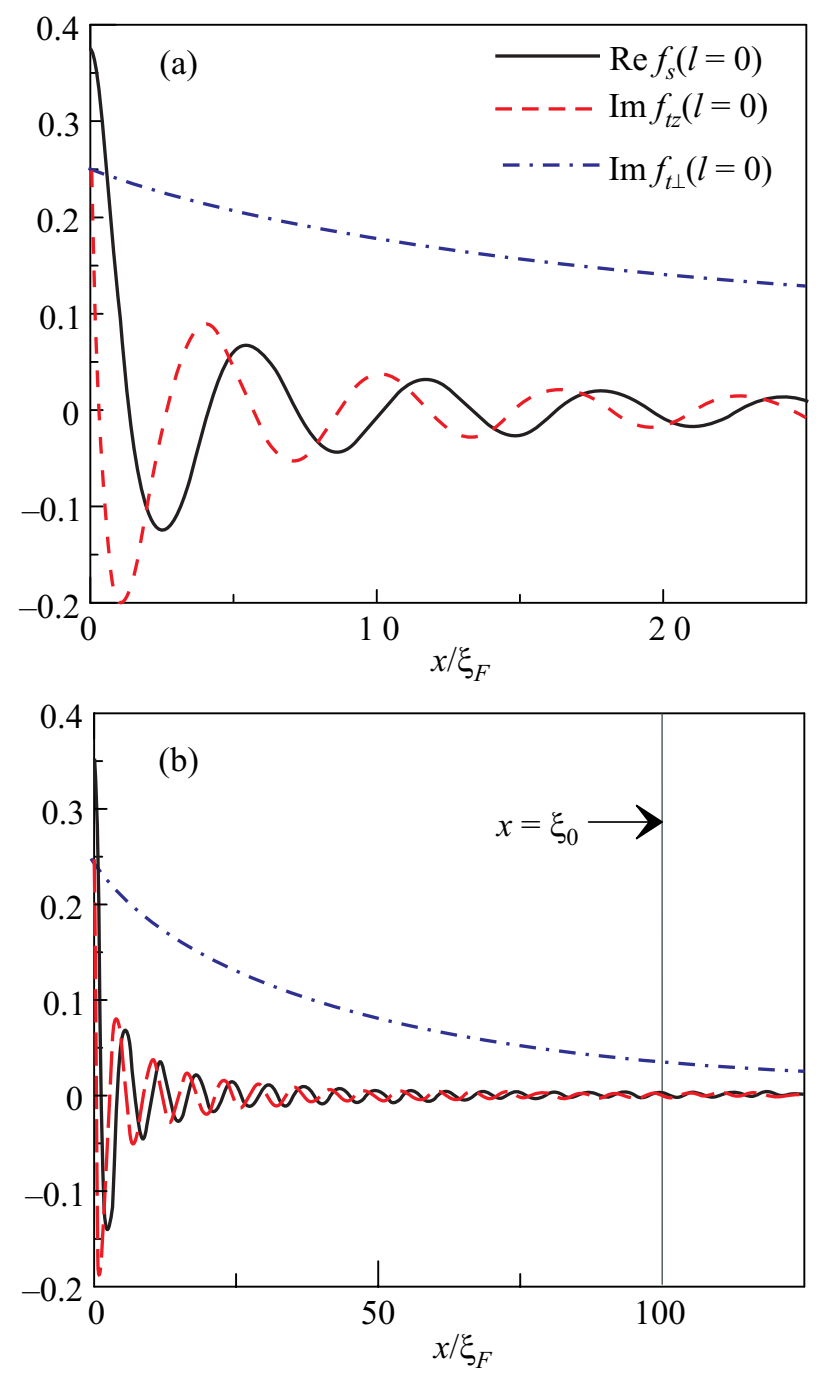

Fig. 8. Pair correlation functions in the ferromagnet of a ballistic $s$-wave superconductor-weak spin-polarized ferromagnet junction. Adopted from Ref. 92. ture near the superconducting critical temperature for the lowest Matsubara frequency, $\omega_{0}=\pi T$. The higher order partial waves $(l \neq 0)$ look very similar and have similar amplitudes. As can be seen in Fig. 8(a), in the region $\xi_{F}<<x<<\xi_{0}=\xi_{T}$ the correlation functions $f_{\uparrow \downarrow}^{S}(x)$ and $f_{\uparrow \downarrow}^{\mathrm{tr}}(x)$ decay like $1 / x$ and are rapidly reduced by a factor $\xi_{F} / \xi_{T}$ (=0.01 for the data in Fig. 8). On the same scale the triplet correlation function with non-zero spin projection $f_{\uparrow \uparrow, \downarrow \downarrow}^{\mathrm{tr}}(x)$ varies smoothly and very slowly. In a much larger distance, $x>\xi_{T}$, Fig. 8(b), all components continue to decay according to $(1 / x) \exp \left(-x / \xi_{T}\right)$. However, the magnitudes of $f_{\uparrow \downarrow}^{S}(x)$ and $f_{\uparrow \downarrow}^{\operatorname{tr}}(x)$ are considerably reduced compared with $f_{\uparrow \uparrow, \downarrow \downarrow}^{\text {tr }}(x)$ before this region is reached. (For more details see Ref. 92.)

The picture changes a little bit in the case of a fully spin polarized ferromagnet. Now the conversion of singlet pairs into triplet ones takes place entirely within the singlet SC. To be definite, we concentrate on an $\mathrm{SSC} / \mathrm{HMF}$ structure suggesting the dirty limit for both metals.

Due to spin mixing at the interfaces, a spin triplet $(S=1$, $m=0)$ amplitude $f_{\uparrow \downarrow}^{\text {tr }}(x)$ is created. This results in a boundary layer with coexisting singlet $f_{\uparrow \downarrow}^{S}(x)$ and triplet $f_{\uparrow \downarrow}^{\mathrm{tr}}(x)$ amplitudes near the interface which extend about a coherence length $\xi_{S}=\left(D_{S} / 2 \pi T\right)^{1 / 2}$ into the SC. Triplet components $f_{\uparrow \uparrow, \downarrow \downarrow}^{\text {tr }}(x)$ are generated if spin-flip centers are present in the interface region. Being created, the equal-spin proximity-induced amplitudes decay slowly in the halfmetallic region on the thermal length scale $\xi_{T}=\left(D_{F} / 2 \pi T\right)^{1 / 2}$. The magnitude of the triplet correlations at the interface is proportional to that of the singlet amplitude at the interface, and both are insensitive to impurity scattering. (More discussion is given in Ref. 92.)

Naturally, the physical mechanisms applicable for $\mathrm{sSC} / \mathrm{HMF}$ heterostructures remain in force for the pointcontact geometry, as well. The impact of spin-mixing and spin-flip AR processes at sSC/HMF interface on PC spectra was theoretically studied just recently in [93,96-99]. It was found that spin-active interface and spin-flip AR can be responsible for the long-range triplet proximity effect. Referring the reader to these reports for a detailed discussion of the problem, we only note here that the authors of Refs. $[93,98,99]$ have proposed an alternative interpretation of the PCAR experimental results that goes beyond the de Jong and Beenakker theory [14]. This alternative interpretation is based on a realistic suggestion that, as already mentioned in Sec. 3.3, it is reasonable to expect that the scattering properties of quasiparticles depend on their spin. In particular, the model utilizes the spin mixing angle $\theta$. It was shown that scattering phase may play an important role in AR process at interfaces to strong ferromagnets. Indeed, the scattering phase difference $\theta$, that quasiparticles incident from the $\mathrm{sSC}$ acquire upon being reflected at $\mathrm{SSC} / \mathrm{HMF}$ interface, induces a triplet Cooper pairs, $f_{\uparrow \downarrow}^{\text {tr }}$, which leads to enhanced subgap conductance. This allows for an alternative interpretation of 
the PCAR spectra, which in this picture can be turned by the fitting parameter $\theta[98,99]$.

It is worth to notice that, when no tunneling potential is present, an inverse magnetic proximity effect $[100,101]$ may affect the $\mathrm{sSC} / \mathrm{F}$ interface transport properties, too.

\subsection{Triplet supercurrent in sSC-manganites PCs}

Let us now go back to the results in Figs. 4-7. If the contact's size is not small, $d>\xi_{S}$, the PE can be important. For proximity affected contacts, it was suggested [25,26,28,29] that the conditions for an unconventional PE are fulfilled. Indeed, the main condition for spin triplet pairing to be induced at $\mathrm{sSC} / \mathrm{F}$ interface is the "spin active" interface, i.e., the ability of the $\mathrm{sSC} / \mathrm{F}$ interface to convert a singlet pair into a triplet one. For manganites, several theoretical models and numerous experimental data, including such local probing as nuclear magnetic resonance [102,103], point that nanoscale nonhomogeneity is an intrinsic feature of these compounds. Another characteristic important for our discussion is that, due to strong Hund's interaction, spin disorder serves as strong spin scattering center for charge carriers. Accordingly, depending on the local magnetic nonhomogeneity at the sSC/doped manganite boundary, the manganites surface causes coherent equal spin $p$-wave even frequency pairing correlations, which spread over large distance into the manganite's bulk [25,26,28,29].

However, the induction of pairing correlations in the normal region is not enough for the realization of multiple AR. As already mentioned, the observation of the subharmonic structure requires the existence of actual gaps in both superconducting electrodes. Thus, experimental finding of the SGS point that the proximity induced superconducting state of manganites possesses an intrinsic superconducting gap $[28,29]$.

Following the physics described, in Fig. 9, the spatial structure of the current through the proximity affected contact is shown. The figure explains mutual conversion of the currents along the contact. In fact, due to a long-range PE, we deal here with a charge transport through an sSC-tSCHMF heterostructure. Namely, there is a region at the $\mathrm{sSC} / \mathrm{HMF}$ interface where a conversion from spin singlet pairs into spin triplet pairs takes place. The equal spin triplet supercurrent flows through the HMF, while the singlet part is completely blocked. The sum of the singlet and triplet currents is constant, obeying the continuity equation. At the boundary of superconducting and normal phases of the manganite a spin polarized supercurrent is continued as a quasiparticle current $j^{q}$ due to the usual AR mechanism. In.deed, at both sides of the $\mathrm{tSC} / \mathrm{HMF}$ interface the charge current is spin polarized and there is no restriction (because of spin) on the AR. As a result, excess current and doubling of the normal-state conductance have to be observed.

The region where transformation of spin singlet pair into spin triplet pairs (and vise verse) takes place is shown in Fig. 10. Figure 10(a) illustrates the so-called "semiconduc-

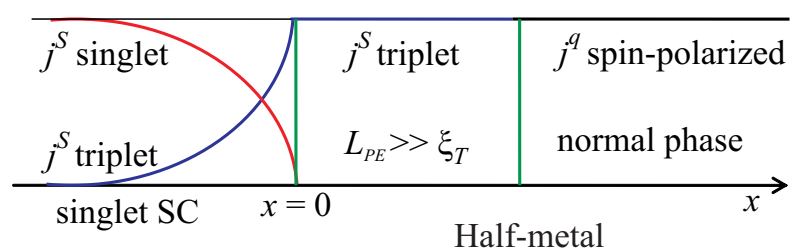

Fig. 9. Spatial structure of the current through the proximity affected $\mathrm{sSC} /$ half-metallic manganite contact. The $x$ axis is directed perpendicular to the sSC/HMF interface that is at $x=0$; the HMF is placed in the region $x>0$, while the sSC is located at $x<0$. Proximity affected region $\mathrm{L}_{P E}$ is much longer than the superconducting coherence length $\xi_{T}=\left(D_{F} / 2 \pi T\right)^{1 / 2}$ and $L_{P E}>\xi_{T}$. Reproduced form Ref. 28.

tor picture" of the proximity affected PC. Figure 10(b) explains the mechanism of conversion between spin singlet and spin triplet pairs due to multiple ARs. At the sSC/tSC interface we deal, in fact, with a weak link (or short constriction) between two different superconductors. The "weak link" here is a region where both singlet and triplet pairing amplitudes are suppressed. In the semiclassical picture, for a given voltage $V<\Delta / e$ across a weak link a quasiparticle accelerated from Fermi surface suffers $n \sim \Delta / e V$ ARs until it reaches the top of the pair potential well. In the particular case shown in Fig. 10(b), an incoming electron/hole of a given spin subband and under the energy gap $\Delta_{S S C}$ cannot enter in the triplet superconducting electrode. It is spin flipped and then Andreev reflected (spin-flip AR process) as a hole/electron back to the $\mathrm{sSC}$, simultaneously adding a triplet Cooper pair to the condensate in the tSC. This hole/electron is spin flipped and then is reflected by Andreev mechanism as an electron/hole back to the tSC, simultaneously adding a singlet Cooper pair to the condensate in the sSC. For a given voltage across the $\mathrm{sSC} / \mathrm{tSC}$ interface a quasiparticle undergoes $n \sim \Delta_{S S C} / \mathrm{V}, \Delta_{t S C} / \mathrm{eV}$, or $m \sim\left(\Delta_{S S C}+\Delta_{t S C}\right) / e V$ reflections (depending on electrodes and energy it starts) until it reaches the top of the pair potential well. As was already indicated, within this physics the subharmonic peaks shown in Figs. 6 and 7 can be specified as it is given in Table 2 .

\subsection{Latent superconductivity of doped manganites}

Let us now make some suggestions concerning the origin of the quasiparticle gap $\Delta_{\operatorname{tr}}$ the magnitude of which cannot be explained in terms of conventional theory of proximity effect.

In mean field BCS-Eliashberg theories with $\Delta(\mathbf{r})=$ $=\left|\Delta_{M F}(\mathbf{r})\right| \exp \{\varphi(\mathbf{r})\}$, the characteristic energy scale responsible for the global transition temperature $T_{C}$ is the superconducting energy gap $\left|\Delta_{M F}(\mathbf{r})\right|$. This silently implies that the spatial variation in $\left|\Delta_{M F}(\mathbf{r})\right|$ is small, and that global phase coherence temperature $T_{\varphi}$ is larger than (or equal to) $T_{C}$. However, for a system with small superfluid densi- 

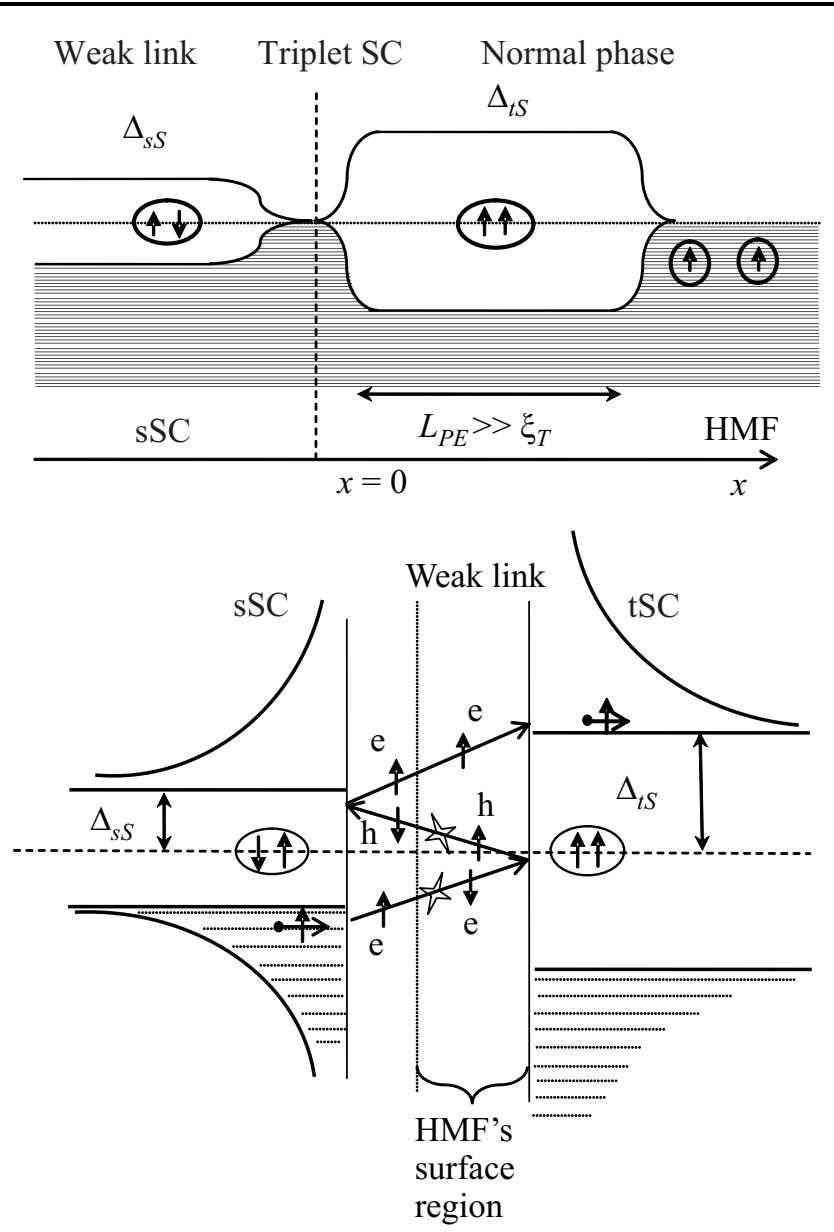

Fig. 10. Semiconductor picture of proximity affected sSC/halfmetallic manganite contact. Weak link here is a region where both singlet and triplet pairing interactions are suppressed (a). Trajectory of a quasiparticle that is accelerated out of the condensate by the electric field suffering multiple Andreev reflections. In the case of singlet and triplet superconducting electrodes every Andreev reflection is foregone with a spin-flip scattering. Spinflip Andreev-reflection processes are illustrated by lines with stars (a). Reproduced form Ref. 29.

ty (bad metal) the spatial variations in the mean-field value $\left|\Delta_{M F}(\mathbf{r})\right|$ could be large. As a consequence, due to large spatial variations, fluctuation effects become crucial in the regions where $\Delta(\mathrm{r})$ is small. Of these, the most important are the thermal fluctuations in the phase of the order parameter $\varphi(\mathbf{r})$. In this case, the fluctuations in the phase of the order parameter in mesoscopic "islands" prevent the long-range superconductivity, i.e., the global critical temperature $T_{C}$ is determined by the global phase coherency, whereas the pair condensate could exist well above $T_{C}[104,105]$.

The important consequence of the presence of the Cooper pair fluctuation above $T_{C}$ is an appearance of the so-called pseudogap $[104,106,107]$ i.e., decreasing of the one-electron density of state near the Fermi level. In particular, according to one point of view [104], in the pseudogap state high- $T_{C}$ cuprates could be considered as an unconventional metal, i.e., as a SC that has lost its phase rigidity due to phase fluctuations. As already mentioned, doped manganites are bad metals. Also, a large pseudogap is indeed detected in numerous experiments on manganites [33-36] and it may be suggested that at least a portion of the observed pseudogap value is due to pairing without global phase coherency. Precursor diamagnetism above $T_{C}$ provides additional arguments for survival of the pair condensate well above $T_{C}$ in cuprates [108,109]. However, for manganites, this kind of response may be strongly suppressed by ferromagnetic order of the localized moments and spin-triplet state of the condensate.

As a likely hypothesis, the authors [28,29] suggested that the results obtained on proximity affected contacts are the observation of a new type of superconducting proximity effect which follows the scenario of a proximity induced superconducting order parameter phase stiffness. Namely, the manganites are thermodynamically very close to a triplet $p$-wave superconducting state and, at low temperature, the local triplet superconducting fluctuations with pairing energy $\Delta_{\text {tr }}$ intrinsically exist ("latent" high- $T_{C}$ superconductivity of doped manganites). Being proximity coupled with singlet superconductor, the phase coherency of a superconducting state is restored.

To verify this phase-disordering scenario for anomalous superconductivity of proximity affected PCs, the authors of a communication [110] prepared and studied normal and superconducting properties of the $\mathrm{MgB}_{2}-$ (nano) $\mathrm{La}_{0.67} \mathrm{Sr}_{0.33} \mathrm{MnO}_{3}$ (MgB:LSMO) composite. The key idea was to obtain such a composite, where proximity affected $\mathrm{HMF} / \mathrm{SC}$ interfaces govern superconducting properties of the bulk sample. Fortunately, the idea was successful: the bulk samples of MgB:LSMO (nano)composite demonstrate direct evidences for unconventional superconductivity. Superconductivity of MgB:LSMO samples with 3:1 and 4:1 weight ratio has been observed with large, up to $20 \mathrm{~K}$, critical temperature. A few features have been detected for bulk samples' characteristics which, most probably, can hardly be explained within the framework of the conventional percolation model. Using the point-contact spectroscopy, three distinct quasiparticle energy gaps $\Delta_{1}(\pi), \Delta_{2}(\sigma)$, and $\Delta_{\text {tr }}$ are clearly revealed. Two of these gaps were identified as enhanced gaps in the quasiparticle spectrum of the $\mathrm{MgB}_{2}$ in the composite; the third gap $\Delta_{\text {tr }}$ was the same as those earlier detected in PCs of $(\mathrm{La}, \mathrm{Sr}) \mathrm{MnO}_{3}$ and (La,Ca) $\mathrm{MnO}_{3}$ with $\mathrm{Pb}$ or $\mathrm{MgB}_{2}$. A noteworthy argument was the temperature behavior of the $\Delta_{\text {tr }}$ gap which did not follow the BCS dependence. The Andreev-reflection spectroscopy on PCs between the samples and half-metallic $\mathrm{La}_{0.65} \mathrm{Ca}_{0.35} \mathrm{MnO}_{3}$ electrode provides an additional evidence in favor of an unconventional superconducting state in the MgB:LSMO composite.

The results obtained assert upon the new type of superconducting proximity effect which provides for the phasecoherency stiffness. At low temperature in a half-metallic ferromagnetic state of $(\mathrm{La}, \mathrm{Sr}) \mathrm{MnO}_{3}$, a phase incoherent 
superconductivity (local triplet pairing condensate) exists. However, though the local gap amplitude is large, there is no phase stiffness and the system is incapable to display long-range superconducting response. Being proximity coupled to $\mathrm{MgB}_{2}$, the long-range coherency is restored. Inversely, the manganite in superconducting state with large energy pairing, due to proximity effect, enhances the $\mathrm{MgB}_{2}$ superconducting state. That is, here we deal with some kind of "mutual" proximity effect.

Naturally, at this stage of investigation, other possible explanations and/or mechanisms, which could be related to the physics of proximity affected PCs of manganites should also be discussed. It was predicted that in $\mathrm{SSC} / \mathrm{F}$ structures, the so-called odd frequency pairing could take place $[89,90,111]$. In this case, the Cooper pair wave function is symmetric under exchange of spatial- and spincoordinates but antisymmetric under exchange of timecoordinates. The study of such pairing in $\mathrm{sSC} / \mathrm{F}$ junction was addressed by a number of authors over the last years [111]. However, if the superconducting correlations are odd in frequency, a pairing interaction has to be additionally frequency dependent (due to strong retardation effect) in order to have a nonzero intrinsic gap in the ferromagnetic metal (see details in Ref. 92).

Nonlocal or crossed AR in which an electron from one magnetic domain is Andreev reflected as a hole into oppositely polarized domain while a pair is transmitted into a superconductor [112-114] is, in principle, possible. However, in order for the data on proximity affected junctions to be in agreement with the crossed AR mechanism, the total current through the contact has to be unpolarized. In may be if the portion of domains with opposite magnetization is exactly equal. It seems improbable that in all the proximity affected PCs the portion of domains with opposite magnetization is exactly equal.

A conversion of spin-less Cooper pair into spin polarized Cooper pair and vice versa is also possible due to absorption (respectively, emission) of a magnon [115]. If this mechanism is governing, the junction's current-voltage characteristic has to be, at low temperatures, asymmetric with respect to the base voltage. The $I(V)$ characteristics of all PCs explored are symmetric, and thus it is hard to suggest that the magnon assisted mechanism controls the charge transport in PCs.

A giant proximity effect (a logarithmic dependence of the junction critical temperature on the junction width) was predicted for a tunnel junction of two SCs with the barrier formed by a $\mathrm{SC}$ that has lost its phase rigidity due to phase fluctuations [116]. We think that to a certain extent this scenario of proximity effect could be relevant to our case.

Extended discussion, including more exotic explanations and/or mechanisms, which could be related to the physics of proximity affected PCs of manganites the reader can find in Ref. [28,29].

\section{Conclusions}

The interplay between superconductivity and spinpolarized materials has potential applications in the emerging field of spin-electronics. Specifically, the so-called superconducting spintronics $[3,117,118]$ is among the most attractive subjects of spintronics, and requires a class of superconducting materials with spin-polarized transport, which would necessarily have to be triplet. The controlled production of triplet supercurrents will open several directions for possible applications. With the availability of fully polarized triplet supercurrents, spin-dependent quantumcoherence phenomena will make implementation of different spintronics devices a reality. Superconducting spintronics devices are appealing since they introduce in a natural way the elements of nonlocality, entanglement, and quantum coherence, all of which are crucial, e.g., for quantum computing. Already existing data [73-82] demonstrate that an effective source of spin-polarized supercurrent can be designed using nanohybrids of metallic ferromagnets and a conventional $s$-wave SCs. Out-of-the-mainstream is the idea that, at low temperature, incoherent superconducting fluctuations are essentially sustained in half-metallic manganites and, in proximity affected region, the singlet superconductor establishes phase coherence of the spinpolarized superconducting state of the manganites $[28,29]$. If it is a success, a promising source of spin-polarized supercurrent can be designed using nano-junctions of manganites and a conventional $s$-wave SC.

Systematic character and repeatability of a number of principal experimental facts suggest that some general physical phenomena have been observed in transport properties of proximity affected singlet superconductor-halfmetallic manganite contacts. Whether the origin of these features can be traced to a thermodynamic state of manganites with local triplet superconducting fluctuations, is a matter of further investigations. Further experiments are definitely needed in order to prove (or disprove) this scenario and understand the mechanism causing the local triplet pairing in doped manganites. We hope that our short review stimulates further experimental and theoretical works on this subject.

1. J.F. Gregg, I. Petej, and C. Dennis, J. Phys. D: Appl. Phys. 35, R121 (2002).

2. A.M. Bratkovsky, Rep. Prog. Phys. 71, 026502 (2008).

3. Y.C. Tao and J.G. Hu, J. Appl. Phys. 107, 041101 (2010).

4. G.-X. Miao, M. Münzenberg, and J.S. Moodera, Rep. Prog. Phys. 74, 036501 (2011).

5. J.-H. Park, E. Vescovo, H.-J. Kim, C. Kwon, R. Ramesh, and T. Venkatesan, Nature (London) 392, 794 (1998).

6. J.M.D. Coey, J.J. Versluijs, and M. Venkatesan, J. Phys. D: Appl. Phys. 35, 2457 (2002).

7. R. Meservey and P.M. Tedrow, Phys. Rep. 238, 173 (1994).

8. A.F. Andreev, Sov. Phys. JETP 19, 1228 (1964).

9. I.K. Yanson, Sov. Phys. JETP 39, 506 (1974). 
10. I.K. Yanson, Fiz. Nizk. Temp. 9, 676 (1983) [Sov. J. Low Temp. Phys. 9, 343 (1983)].

11. I.K. Yanson, and O.I. Shklyarevskii, Fiz. Nizk. Temp. 12, 885 (1986) [Sov. J. Low Temp. Phys. 12, 509 (1986)].

12. Y.G. Naidyuk and I.K. Yanson, Point-Contact Spectroscopy, Springer (2005).

13. D. Daghero and R.S. Gonelli, Supercond. Sci. Technol. 23, 043001 (2010).

14. M.J.M. de Jong and C.W. Beenakker, Phys. Rev. Lett. 74, 1657 (1995).

15. R.J. Soulen, Jr., J.M. Byers, M.S. Osofsky, B. Nadgorny, T. Ambrose, S.F. Cheng, P.R. Broussard, C.T. Tanaka, J. Nowak, J.S. Moodera, A. Barry, and J.M.D. Coey, Science 282, 85 (1998).

16. S.K. Upadhyay, A. Palanisami, R.N. Louie, and R.A. Buhrman, Phys. Rev. Lett. 81, 3247 (1998).

17. G.J. Strijkers, Y. Ji, F.Y. Yang, C.L. Chien, and J.M. Byers, Phys. Rev. B 63, 104510 (2001).

18. B. Nadgorny, R.J. Soulen, Jr., M.S. Osofsky, I.I. Mazin, G. Laprade, R.J.M. van de Veerdonk, A.A. Smits, S.F. Cheng, E.F. Skelton, and S.B. Qadri, Phys. Rev. B 61, R3788 (2000).

19. R.J. Soulen, Jr., M.S. Osofsky, B. Nadgorny, T. Ambrose, P. Broussard, S.F. Cheng, J. Byers, C.T. Tanaka, J. Nowack, J.S. Moodera, G. Laprade, A. Barry, and M.D. Coey, J. Appl. Phys. 85, 4589 (1999).

20. Y. Ji, G.J. Strijkers, F.Y. Yang, and C.L. Chien, Phys. Rev. B 64, 224425 (2001).

21. J.S. Parker, S.M. Watts, P.G. Ivanov, and P. Xiong, Phys. Rev. Lett. 88, 196601 (2002).

22. B. Nadgorny, I.I. Mazin, M. Osofsky, R.J. Soulen, Jr., P. Broussard, R.M. Stroud, D.J. Singh, V.G. Harris, A. Arsenov, and Y. Mukovskii, Phys. Rev. B 63, 184433 (2001).

23. Y. Ji, C.L. Chien, Y. Tomioka, and Y. Tokura, Phys. Rev. B 66, 012410 (2002).

24. G.T. Woods, R.J. Soulen, Jr., I. Mazin, B. Nadgorny, M.S. Osofsky, J. Sanders, H. Srikanth, W.E. Egelhoff, and R. Datla, Phys. Rev. B 70, 054416 (2004).

25. V.N. Krivoruchko, V.Yu. Tarenkov, A.I. D'yachenko, and V.N. Varyukhin, Europhys. Lett. 75, 294 (2006).

26. A.I. D'yachenko, V.N. Krivoruchko, and V.Yu. Tarenkov, Fiz. Nizk. Temp. 32, 1085 (2006) [Low Temp. Phys. 32, 824 (2006)].

27. A.I. D'yachenko, V.A. D'yachenko, V.Yu. Tarenkov, and V.N. Krivoruchko, Phys. Solid State 48, 432 (2006).

28. V.N. Krivoruchko and V.Yu. Tarenkov, Phys. Rev. B 75, 214508 (2007).

29. V.N. Krivoruchko and V.Yu. Tarenkov, Phys. Rev. B 78, 054522 (2008).

30. I. Leonov, A.N. Yaresko, V.N. Antonov, and V.I. Anisimov, Phys. Rev. B 74, 165117 (2006).

31. Yu.S. Dedkov, U. Rüdiger, and G. Güntherodt, Phys. Rev. B 65, 064417 (2002).

32. P.Y. Song, J.F. Wang, C.P. Chen, H. Deng and Y.D. Li, J. Appl. Phys. 100, 044314 (2006).
33. E. Dagotto, T. Hotta, and A. Moreo, Phys. Rep. 344, 1 (2001).

34. M.B. Salamon and M. Jaime, Rev. Mod. Phys. 73, 583 (2001).

35. V.M. Loktev and Yu.G. Pogorelov, Fiz. Nizk. Temp. 26, 231 (2000) [Low Temp. Phys. 26, 171 (2000)].

36. J.M.D. Coey, M. Viret, and S. von Molnar, Adv. Phys. 48, 167 (1999).

37. V.N. Krivoruchko and S.I. Khartsev, Fiz. Nizk. Temp. 24, 1070 (1998) [Low Temp. Phys. 24, 803 (1998)].

38. W.E. Pickett and D.J. Singh, Phys. Rev. B 53, 1146 (1996).

39. S. Satpathy, Z.S. Popovic, and F.R. Vukajlovic, Phys. Rev. Lett. 76, 960 (1996).

40. W.J. Chang, J.Y. Tsai, H.-T. Jeng, J.-Y. Lin, Kenneth Y.-J. Zhang, H.L. Liu, J.M. Lee, J.M. Chen, K.H. Wu, T.M. Uen, Y.S. Gou, and J.Y. Juang, Phys. Rev. B 72, 132410 (2005).

41. Q. Zhang and W. Zhang, Phys. Rev. B 68, 134449 (2000).

42. Ch. Ma, Zh. Yang, and S. Picozzi, J. Phys. Condens. Matter. 8, 7717 (2006).

43. I. Golosov, Phys. Rev. Lett. 104, 207207 (2010).

44. J.Y.T. Wei, N.-C. Yeh, and R.P. Vasquez, Phys. Rev. Lett. 79, 5150 (1997).

45. Y. Okimoto, T. Katsufuji, T. Ishikawa, A. Urushibara, T. Arima, and Y. Tokura, Phys. Rev. Lett. 75, 109 (1995).

46. R. Bertacco, M. Portalupi, M. Marcon, L. Duó, F. Ciccacci, M. Bowen, J.P. Contour, and A. Barthélémy, J. Magn. Magn. Mater. 242-245, 710 (2002).

47. S.W. Han, J.-S. Kang, K.H. Kim, J.D. Lee, J.H. Kim, S.C. Wi, C. Mitra, P. Raychaudhuri, S. Wirth, K.J. Kim, B.S. Kim, J.I. Jeong, S.K. Kwon, and B.I. Min, Phys. Rev. B 69, 104406 (2004).

48. A. Chikamatsu, H. Wadati, H. Kumigashira, M. Oshima, A. Fujimori, N. Hamada, T. Ohnishi, M. Lippmaa, K. Ono, M. Kawasaki, and H. Koinuma, Phys. Rev. B 73, 195105 (2006).

49. H.Y. Hwang, S-W. Cheong, N.P. Ong, and B. Batlogg, Phys. Rev. Lett. 77, 2041 (1996).

50. J.Z. Sun, W.J. Gallagher, P.R. Duncombe, L. KrusinElbaum, R.A. Altman, A. Gupta, Yu Lu, G.Q. Gong, and Gang Xiao, Appl. Rev. Lett. 69, 3266 (1996).

51. Y. Lu, X.W. Li, G.Q. Gong, G. Xiao, A. Gupta, P. Lecoeur, J.Z. Sun, Y.Y. Wang and V.P. Dravid, Phys. Rev. B 54, R8357 (1996).

52. J. Z. Sun, L. Krusin-Elbaum, P.R. Duncombe, A. Gupta, and R.B. Laibowitz, Appl. Phys. Lett. 70, 1769 (1997).

53. D.C. Worledge and T.H. Geballe, Appl. Phys. Lett. 76, 900 (2000).

54. Moon-Ho Jo, N.D. Mathur, N.K. Todd, and M.G. Blamire, Phys. Rev. B 61, 14905 (2000).

55. M. Bowen, M. Bibes, A. Barthélémy, J.-P. Contour, A. Anane, Y. Lemaître, and A. Fert, Appl. Phys. Lett. 82, 233 (2003).

56. I.I. Mazin, Phys. Rev. Lett. 83, 1427 (1999).

57. I.I. Mazin, A.A. Golubov, and B. Nadgorny, J. Appl. Phys. 89, 7576 (2001).

58. G. Wexler, Proc. Phys. Soc. 89, 927 (1966).

59. A.I. Buzdin, Rev. Mod. Phys. 77, 935 (2005). 
60. A.V. Zaitsev, Zh. Eksp. Teor. Fiz. 78, 221 (1980).

61. G.E. Blonder, M. Tinkham, and T.M. Klapwijk, Phys. Rev. B 25, 4515 (1982).

62. Yu.V. Sharvin, Sov. Phys. JETP 21, 655 (1965).

63. C.H. Kant, O. Kurnosikov, A.T. Filip, P. LeClair, H.J.M. Swagten, and W.J.M. de Jonge, Phys. Rev. B 66, 212403 (2002).

64. P. Chalsani, S.K. Upadhyay, O. Ozatay, and R.A. Buhrman, Phys. Rev. B 75, 094417 (2007).

65. A. Martín-Rodero, A. Levy Yeyati, and J.C. Cuevas, Physica C 353, 67 (2001).

66. F. Pérez-Willard, J.C. Cuevas, C. Surgers, P. Pfundstein, J. Kopu, M. Eschrig, and H.V. Lohneysen, Phys. Rev. B 69, 140502 (2004).

67. R.C. Dynes, V. Narayanamurti, and J.P. Garno, Phys. Rev. Lett. 41, 1509 (1978).

68. E.L. Wolf, Principles of Electron Tunneling Spectroscopy, Oxford University Press, New York (1985).

69. U. Gunsenheimer and A.D. Zaikin, Phys. Rev. B 50, 6317 (1994).

70. M. Hurd, S. Datta, and P.F. Bagwell, Phys. Rev. B 54, 6557 (1996).

71. B.A. Aminov, A.A. Golubov, and M.Yu. Kupriyanov, Phys. Rev. B 53, 365 (1996).

72. X.X. Xi, Rep. Prog. Phys. 71, 116501 (2008).

73. M. Giroud, H. Courtois, K. Hasselbach, D. Mailly, and B. Pannetier, Phys. Rev. B 58, R11872 (1998).

74. V.T. Petrashov, I.A. Sosnin, I. Cox, A. Parsons, and C. Troadec, Phys. Rev. Lett. 83, 3281 (1999).

75. M.D. Lawrence and N. Giordano, J. Phys. Condens. Matter 11, 1089 (1999).

76. V. Peňa, Z. Sefrioui, D. Arias, C. Leon, J. Santamaria, M. Varela, S.J. Pennycook, and J.L. Martinez, Phys. Rev. B 69, 224502 (2004).

77. I. Sosnin, H. Cho, V.T. Petrashov, and A.F. Volkov, Phys. Rev. Lett. 96, 157002 (2006).

78. R.S. Keizer, S.T.B. Goennenwein, T.M. Klapwijk, G. Maio, G. Xiao, and A. Gupta, Nature (London) 439, 825 (2006).

79. T.S. Khaire, M.A. Khasawneh, W.P. Pratt, Jr., and N.O. Birge, Phys. Rev. Lett. 104, 137002 (2010).

80. J. Wang, M. Singh, M. Tian, N. Kumar, B. Liu, C. Shi, J.K. Jain, N. Samarth, T.E. Mallouk, and M.H.W. Chan, Nature Phys. 6, 389 (2010).

81. Y. Kalcheim, O. Millo, M. Egilmez, J.W.A. Robinson, and M.G. Blamire, Phys. Rev. B 85, 104504 (2012).

82. C. Klose, T.S. Khaire, Yixing Wang, W.P. Pratt, Jr., N.O. Birge, B.J. McMorran, T.P. Ginley, J.A. Borchers, B.J. Kirby, B.B. Maranville, and J. Unguris, Phys. Rev. Lett. 108, 127002 (2012).

83. M. Kasai, T. Ohno, Y. Kanke, Y. Kozono, M. Hanazono, and Y. Sugita, Jpn. J. Appl. Phys. 29, L2219 (1990).

84. M. Kasai, Y. Kanke, T. Ohno, and Y. Kozono, J. Appl. Phys. 72, 5344 (1992).

85. J. Hoppler, J. Sthan, Ch. Nidermayer, V.K. Malik, H. Boyanfif, A.J. Drew, M. Rossle, A. Buzdin, G. Cristiani,
H.U. Habermeier, B. Keimer, and C. Bernhard, Nature Mater. 8, 315 (2009).

86. Z. Sefrioui, D. Arias, V. Pena, J.E. Villegas, M. Varela, P. Prieto, C. Leon, J.L. Martinez, and J. Santamaria, Phys. Rev. $B$ 67, 214511 (2003).

87. K. Dybko, K. Werner-Malento, P. Aleshkevych, M. Wojcik, M. Sawicki, and P. Przyslupski, Phys. Rev. B 80, 144504 (2009).

88. Y. Kalcheim, T. Kirzhner, G. Koren, and O. Millo, Phys. Rev. B 83, 064510 (2011).

89. A. Kadigrobov, R.I. Skekhter, and M. Jonson, Europhys. Lett. 54, 394 (2001)

90. F.S. Bergeret, A.F. Volkov, and K.B. Efetov, Phys. Rev. Lett. 86, 4096 (2001).

91. M. Eschrig, J. Kopu, J.C. Cuevas, and G. Schön, Phys. Rev. Lett. 90, 137003 (2003).

92. M. Eschrig and T. Löfwander, T. Champel, J. C. Cuevas, J. Kopu, and G. Schön, J. Low Temp. Phys. 147, 457 (2007).

93. R. Grein, T. Löfwander, G. Metalidis, and M. Eschrig, Phys. Rev. B 81, 094508 (2010).

94. A.A. Abrikosov, L.P. Gorkov, and I.E. Dzyaloshinski, Methods of Quantum Field Theory in Statistical Physics, Prentice-Hall, Englewood Cliffs NJ (1963).

95. T. Tukuyasu, J.A. Sauls, D. Rainer, Phys. Rev. B 38, 8823 (1988).

96. J.N. Kupferschmidt and P.W. Brouwer, Phys. Rev. B 83, 014512 (2011).

97. F.B. Wilken and P.W. Brouwer, Phys. Rev. B 85, 134531 (2012).

98. T. Löfwander, R. Grein, and M. Eschrig, Phys. Rev. Lett. 105, 207001 (2010).

99. S. Piano, R. Grein, C.J. Mellor, K. Výborný, R. Campion, M. Wang, M. Eschrig, and B.L. Gallagher, Phys. Rev. B 83, 081305(R) (2011).

100. V.N. Krivoruchko and E.A. Koshina, Phys. Rev.B 66, 014521 (2002).

101. F.S. Bergeret, A.F. Volkov, and K.B. Efetov, Phys. Rev. B 69, 174504 (2004).

102. M.M. Savosta, V.N. Krivoruchko, I.A. Danielenko, V.Y. Tarenkov, T.E. Konstantinova, A.V. Borodin, and V.N. Varyukhin, Phys. Rev. B 69, 024413 (2004)

103. A.S. Mazur, V.N. Krivoruchko, and I.A. Danilenko, Fiz. Nizk. Temp. 33, 1227 (2007) [Low Temp. Phys. 33, 931 (2007)].

104. V.J. Emery and S.A. Kivelson, Phys. Rev. Lett. 74, 3253 (1995).

105. E. Berg, D. Orgad, and S.A. Kivelson, Phys. Rev. B 78, 094509 (2008).

106. V.M. Loktev, R.M. Quick, and S.G. Sharapov, Phys. Rep. 349, 1 (2001).

107. M. Norman, D. Pines, and C. Kallin, Adv. Phys. 54, 715 (2005).

108. Lu Li, Y. Wang, S. Omiya, S. Ono, Y. Ando, G.D. Gu, and N.P. Ong, Phys. Rev. B 81, 054510 (2010).

109. J. Mosqueira, J.D. Dancausa, and F. Vidal, Phys. Rev. B 84, 174518 (2011). 
110. V.N. Krivoruchko and V.Y. Tarenkov, Phys. Rev. B 86, 104502 (2012).

111. F.S. Bergeret, A.F. Volkov, and K.B. Efetov, Rev. Mod. Phys. 77, 1321 (2005).

112. G. Deutscher and D. Feinberg, Appl. Phys. Lett. 76, 487 (2000).

113. D. Beckmann, H.B. Weber, and H. v. Lőhneysen, Phys. Rev. Lett. 93, 197003 (2004).

114. P. Aronov and G. Koren, Phys. Rev. B 72, 184515 (2005).

115. G. Tkachov, E. McCann, and V. Fal'ko, Phys. Rev. B 65, 024519 (2001).
116. D. Marchand, L. Covaci, M. Berciu, and M. Franz, Phys. Rev. Lett. 101, 097004 (2008).

117. S. Anders, M.G. Blamire, F.-Im. Buchholz, D.-G. Crété, R. Cristiano, P. Febvre, L. Fritzsch, A. Herr, E. Il'ichev, J. Kohlmann, J. Kunert, H.-G. Meyer, J. Niemeyer, T. Ortlepp, H. Rogalla, T. Schurig, M. Siegel, R. Stolz, E. Tarte, H.J.M. ter Brake, H. Toepfer, J.-C. Villegier, A.M. Zagoskin, and A.B. Zorin, Physica C 470, 2079 (2010).

118. M. Eschrig, Phys. Today 64, 43 (2011). 